Final Report on NASA Grant No. NAG 3-919

\title{
A Prediction of 3-D Viscous Flow and Performance of the NASA Low-Speed Centrifugal Compressor
}

\author{
by
}

John Moore

Professor of Mechanical Engineering

Principal Investigator and

Joan G. Moore

Research Associate

January 1989

Grantee Institution

NASA Lewis Research Center

21000 Brookpark Road

Cleveland, Ohio 44135

Turbomachinery Research Group

Report No. JM/89-1

Mechanical Engineering Department

Virginia Polytechnic Institute and State University

Blacksburg, Virginia 24061-0238 


\section{ABSTRACT}

A prediction of the three-dimensional turbulent flow in the NASA Low-Speed Centrifugal Compressor Impeller has been made. The calculation was made for the compressor design conditions with the specified uniform tip clearance gap. The predicted performance is significantly worse than that predicted in the NASA design study. This is explained by the high tip leakage flow in the present calculation and by the different model adopted for tip leakage flow mixing. The calculation gives an accumulation of high losses in the shroud/pressure-side quadrant near the exit of the impeller. It also predicts a region of meridlonal backflow near the shroud wall. Both of these flow features should be extensive enough in the NASA impeller to allow detailed flow measurements, leading to 1mproved flow modelling. Recommendations are made for future flow studies in the NASA impeller. 
ABSTRACT

NOMENCLATURE. $\ldots \ldots \ldots \ldots \ldots \ldots \ldots \ldots \ldots \ldots \ldots \ldots \ldots \ldots \ldots \ldots \ldots \ldots \ldots \ldots \ldots \ldots \ldots \ldots \ldots \ldots \ldots \ldots$

INTRODUCTION. .........................................

NASA Low-Speed Centrifugal Compressor Research Program

NASA Low-Speed Centrifugal Compressor

Contribution of Present Work

COMPUTATIONAL METHOD................................

Use of Support Calculations

COMPRESSOR GEOMETRY AND COMPUTATIONAL GRIDS...................8

Meridional Flow Calculation

3-D Flow Calculation

RESULTS OF MERIDIONAL FLOW CALCULATION...................... 10

TEST OF GRID SELECTED FOR TIP GAP........................1

RESULTS OF 3-D FLOW CALCULATION.........................11

Meridional Flow

Straight-Walled Vaneless Diffuser

Modifled Vaneless Diffuser

Thermodynamic Performance...........................12

Impeller and Compressor Efficiencies

Work Input

Static Temperature Changes in Impeller

Total Temperature Changes in Impeller

3-D Pressure Distribution............................ 18

Impeller Blade Loading

Blade Surfaces

Hub Wall and Blade Tip Region

Shroud Static Pressure Distribution

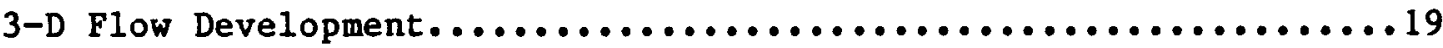

Blade-to-Blade Planes

Cross-Sectional Planes

Detail of Flow Through Tip Gap

Cumulative Flow Through Tip Gap

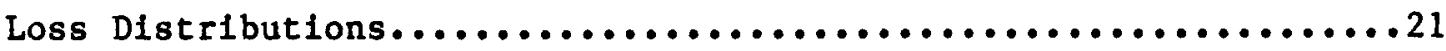

DISCUSSION OF RESULTS.................................. 22

Tip Leakage Flow Mixing

Jet-Wake Mixing

Backflow Along Impeller Shroud Wall 
Table of Contents (Cont'd)

Page

Comparison with NASA Flow and Performance Predictions

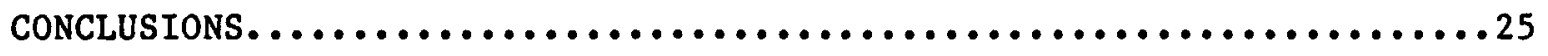

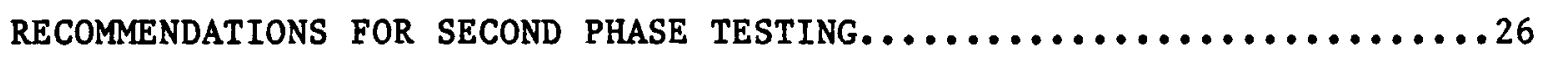

RECOMMENDATIONS FOR THIRD PHASE TESTING.....................26

REFERENCES........................................... 27

FIGURES. $\ldots \ldots \ldots \ldots \ldots \ldots \ldots \ldots \ldots \ldots \ldots \ldots \ldots \ldots \ldots \ldots \ldots \ldots \ldots \ldots \ldots \ldots \ldots \ldots \ldots \ldots$ 


\section{NOMENCLATURE}

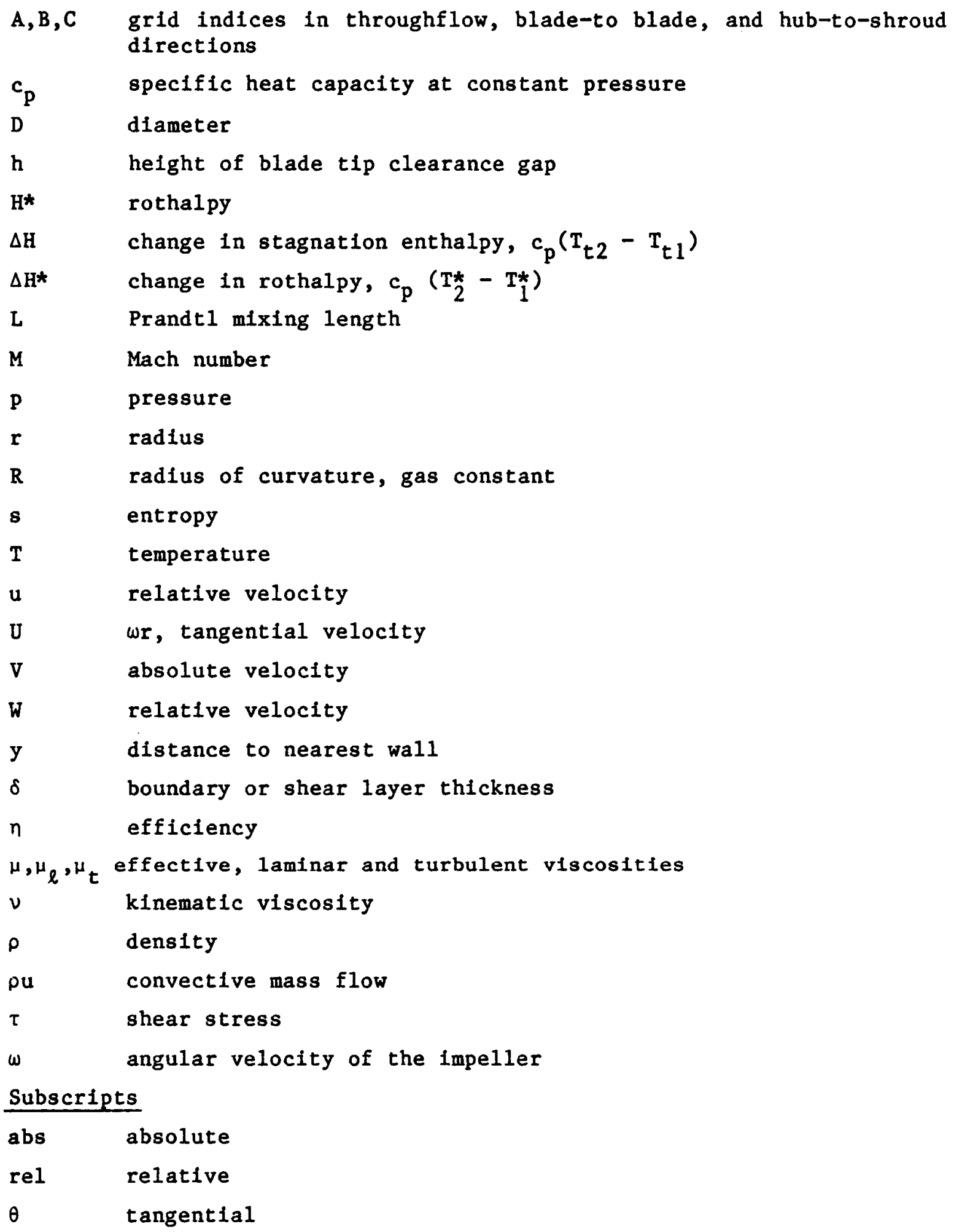




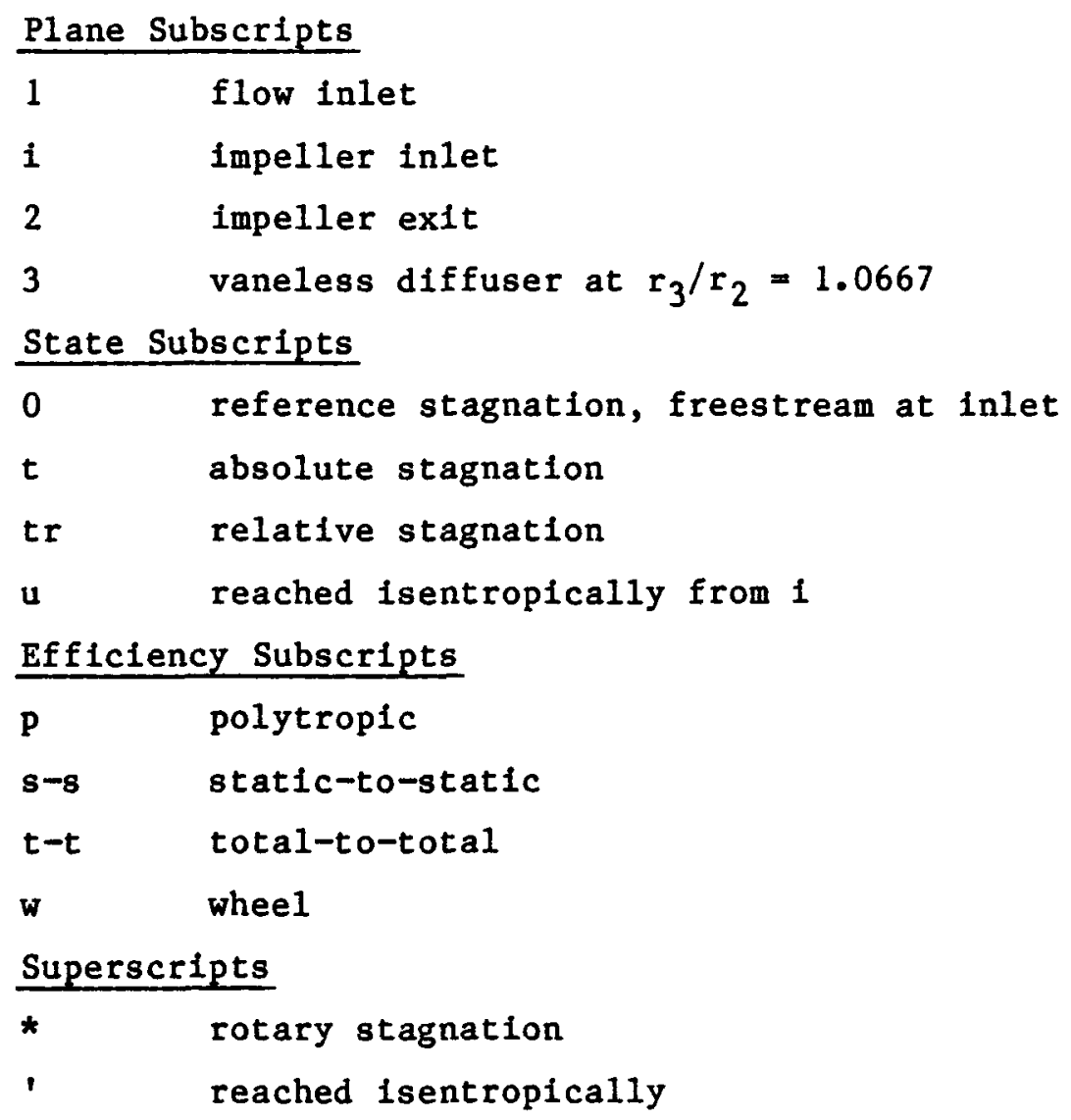




\section{INTRODUCTION}

MASA Low-Speed Centrifugal Compressor Research Progran [1]

The new low-speed centrifugal compressor facility at NASA Lewis Research Center has been bullt to enable a more thorough understanding of subsonic flow in the complex geometric channels of centrifugal compressors to be obtained. NASA is planning an experimental program to obtaln "benchmark" experimental data to verify three-dimensional viscous flow codes and to provide data with which to develop more sophisticated models of the various physical phenomena occuring in a centrifugal compressor. The complex phenomena to be considered include secondary flows generated by inlet vorticity, secondary flows generated when boundary layers are subjected to transverse forces, tip clearance flows, and turbulence suppression and enhancement due to strong normal pressure gradients occuring across the boundary layers in the impeller passage.

\section{MASA Low-Speed Centrifugal Compressor [1]}

The configuration of the compressor is shown in Fig. 1. The 1mpeller has an exit diameter of $1.524 \mathrm{~m}$ (60 inches) and an inlet hub/tip ratio of 0.5 . There are 20 full impeller blades with an exit backsweep of $55^{\circ}$. The design speed of 1920 RPM gives a rotor exit tip speed of $153 \mathrm{~m} / \mathrm{s}(503 \mathrm{ft} / \mathrm{s})$. The design mass flow rate is $30 \mathrm{~kg} / \mathrm{s}(66 \mathrm{lbm} / \mathrm{s})$ at the standard inlet conditions of $101,325 \mathrm{~N} / \mathrm{m}^{2}$ and $288.15 \mathrm{~K}$. The rotor tip Reynolds number $\left(\mathrm{U}_{2} \mathrm{D}_{2} / \nu_{0}\right)$ is $16 \times 10^{6}$. Initially the compressor will be run with a vaneless diffuser.

The nominal setting of the rotor tip clearance is a uniform $5.7 \mathrm{~mm}$ $(0.225$ inch). This tip clearance is 2.6 percent of the hub-shroud blade height at the Impeller inlet and 4.0 percent of the blade height at the exit.

The design philosophy adopted for the impeller was to obtain smooth velocity distributions while maintaining minimum blade lean. The preliminary design code used the Katsanis and McNally meridional flow program [2] for flow analysis. Velocity distributions were then calculated using a three-dimensional Euler code as described by Denton in 
reference 3. Figure 2 shows the velocity triangles and blade loading resulting from this preliminary design procedure. The rotor inlet tip Mach number is 0.31 ; the blade loading is quite unfform throughout the impeller; and the predicted relative Mach number at the Impeller exit is apparently non-uniform, varying from 0.2 at the tip to 0.25 at the hub. The rotor exit absolute Mach number is 0.29 .

The NASA predictions of rotor total pressure ratio and (total-tototal) efficiency are then 1.173 and 0.934 , respectively. The work input parameter $\Delta \mathrm{H} / \mathrm{U}^{2}$ is anticipated to be 0.607 .

\section{Contribution of Present Work}

The objective of this study was to perform a three-dimensional turbulent flow calculation for the NASA Compressor at its design operating conditions. Similar calculations were recently performed by the authors for the earlier NACA 48-Inch Radial-Inlet Centrifugal Impeller $[4,5]$. Those calculations reproduced the measured overall performance and most of the features of the loss distributions in the NACA flow study. They gave further insight into the complex 3-D flow with tip leakage. It is therefore hoped that the present 3-D flow calculation for the NASA rotor will provide a useful prediction to ald in planning experimental work and flow measurements.

For example, the preliminary 2-D boundary layer calculations of Wood et al. [1] suggested boundary layer thicknesses of approximately one inch $(2.54 \mathrm{~cm})$ near the NASA rotor exit. The present calculation shows regions of thicker boundary layer flow and loss accumulation where measurements may be easier, and also regions of thin boundary layer flow where measurements may be more difficult. In addition, the calculation includes a stationary shroud wall and a detafled representation of flow through the tip gap. This allows the shroud boundary layer development, including the infuence of tip leakage flow mixing, to be predicted; and the calculation shows regions of reverse flow in the meridional direction. The development of the shroud boundary layer appears to strongly influence both the impeller and the diffuser performance. 
The calculation results suggest flow mechanisms which are critical to the flow development and performance of this compressor. They demonstrate another level of preliminary flow calculation and prediction, for comparison with the results of the NASA preliminary design calculations made for this compressor. The results also allow specific suggestion for changes in the impeller and diffuser geometries to improve the performance of the compressor to be made.

\section{COMPUTATIONAL METHOD}

The calculation was performed with the Moore Elliptic Flow Program, a 3-D pressure-correction solution procedure for discretized forms of the Navier-Stokes equations. The conservation equations for mass, momentum and energy used for steady compressible flow are shown in Table 1. The discretization of the conservation equations is described in reference 6 . The equations are solved using the solution procedure described in reference 7.

A Prandtl mixing-length turbulence model, summarized in Table 2, was used. For this calculation, a free-stream mixing length of 0.000387 $m$ was chosen, corresponding to an estimate of 0.5 percent free-stream turbulence at the rotor inlet.

Use of Support Calculations

Two preliminary 2-D flow calculations were performed:

1. A meridional flow calculation was used to obtain inlet conditions for the 3-D flow calculation.

2. A tip gap flow calculation was used to test the computational mesh chosen around the blade tip.

\section{COMPRESSOR GEOMETRY AND COMPUTATIONAL GRIDS}

\section{Meridional Flow Calculation}

The inlet bellmouth, the inlet duct and the shroud contour of the impeller are shown in Fig. 3. 2-D meridional flow through the impeller was modelled using a $40 \times 2 \times 22$ grid, shown in Fig. 4; this included the rotating spinner and hub of the impeller. 
Table 1. Steady Flow Conservation Equations

Mass

$$
\nabla \cdot p \underline{u}=0
$$

Momentum

$\rho \underline{u} \cdot \nabla \underline{u}-(\nabla \cdot \mu \nabla) \underline{u}=\nabla \cdot \mu \overline{\nabla \underline{u}}^{T}-\nabla p-2 \rho \underline{\omega} \times \underline{u}-\rho \underline{\omega} \times(\underline{\omega} \times \underline{I})$

Equation of State

$$
p=\rho R T
$$

Rothalpy (energy)

$$
\begin{aligned}
& \rho \underline{u} \cdot \nabla H^{*}-(\nabla \cdot \mu \nabla) H^{*}=0 \\
& H^{*} \equiv c_{p} I+\frac{1}{2}(\underline{u} \cdot \underline{u})-\frac{I}{2}(\underline{\omega} \times \underline{I} \cdot \underline{\omega} \times \underline{I})
\end{aligned}
$$

Table 2. Prandtl Mixing Length Viscosity Model

$$
\begin{aligned}
& \mu=\mu_{\ell}+\mu_{t} \\
& \mu_{t}=\rho L^{2} " \frac{d u^{\prime}}{d y}
\end{aligned}
$$

$L$ is the smaller of

0.08 times width of shear or boundary layer

0.41 times distance to nearest wall

Van Driest Correction

$$
L=0.41 " y "\left(1 .-\exp \left[-" y " \sqrt{\rho \tau} / 26 \mu_{\ell}\right]\right)
$$

Near-Wa11 Correction

$$
\mu=\sqrt{\mu_{\ell}\left(\mu_{\ell}+\mu_{t}\right)} .
$$


The $53 \times 21 \times 22$ grid for the 3-D flow calculation is shown in Figs. 5, 6, and 7. Figure 4 shows the inlet for the 3-D flow calculation $0.188 \mathrm{~m}$ ( 7.4 inches) upstream of the impeller inlet, part way along the spinner; this is the start of the grids in Fig. 5. The calculation was performed through the vaneless diffuser to a radius ratio $r / r_{2}=$ 1.5. Figure 5a shows the basic constant-height vaneless diffuser; this was redesigned during the calculation to have a constant area starting at $r / r_{2}=1.08$, as seen in Fig. $5 \mathrm{~b}$.

Blade-to-blade sections of the 3-D grid are shown in Fig. 6 . The 55 degree backsweep is evident in the shroud and hub sections in Figs. $6 \mathrm{a}$ and 6b, and leading and trailing edge detail is seen in Figs. 6c and 6d. Cross sections of the grid at the impeller exit and near the impeller inlet, shown in Fig. 7, illustrate the grid refinement used around the blade tip and the walls of the impeller passage.

\section{RESULTS OF RERIDIONAL FLON CALCULATION}

In the 2-D meridional flow calculation, turbulent boundary layers were started on the shroud wall $1.04 \mathrm{~m}$ (41 inches) upstream of the location of the impeller leading edge and at the stagnation point on the spinner. The flow then developed as shown in Fig. 8. By the inlet for the 3-D flow calculation, the shroud wall boundary layer thickness was about 10 percent of the local passage height while the boundary layer on the rotating spinner was about 3 percent thick, as shown in Fig. 8c. The velocity vectors in Fig. 8a show separation of the shroud boundary layer near the impeller exit as the static pressure on the shroud wall recovers from the minimum at about 30 percent of meridional distance through the impeller, seen in Fig. 8b. At the exit of the flow domain, at $r / r_{2}=1.5$, the shroud wall boundary layer fills the diffuser but the negative mass flow is only about 0.7 percent of the total. 


\section{TEST OF GRID SELECTED FOR TIP GAP}

A two-dimensional tip gap/cavity geometry was set up to test the grid spacing within and around the blade tip. The ratio of the circumferential length to the height of the tip clearance gap varies from about one near the impeller leading edge to about two at the impeller exit. Figure 9 shows results for flow through a square tip gap of height $h$ entering a cavity of overall height $10 \mathrm{~h}$ and width $9 \mathrm{~h}$. A typical Reynolds number of 24,000 , based on the mean velocity in the tip gap and the height $h$, was chosen.

The velocity vectors in Figs. $9 a$ and $9 \mathrm{c}$ also show the locations of the grid points. Overall, a $27 \times 12 \times 2$ grid was used, with four grid points in the flow path between the blade tip and an inviscid shroud wall. The near-wall points are located $0.1 \mathrm{~h}$ from the blade tip; and with such a coarse grid there is no evidence of the expected flow separation at the sharp tip gap entrance. Indeed, although the flow distribution at the tip gap exit in Fig. 9c seems qualitatively reasonable, it probably represents a tip leakage flow about 10 percent too large, due to an effective rounding of the inlet corner [8].

After leaving the tip gap, the flow mixes and spreads as it moves across the endwall, see Fig. 9b. It then splits as it encounters the cavity exit, with some of the flow circulating around the cavity. These same general features are probably superposed on the throughflow in the impeller.

\section{RESULTS OF 3-D FLOW CALCULATION}

\section{Meridional Flov}

\section{Straight Walled Vaneless Diffuser}

The 3-D flow calculation was started with the straight-walled vaneless diffuser. Figure 10a, however, shows that this resulted in strong backflow, in excess of 10 percent of the throughflow, at the diffuser exit. This violated the exit boundary condition. So the diffuser was redesigned to have a constant area from a radius ratio $\mathrm{r} / \mathrm{r}_{2}$ $=1.08$, 1.e. from the leading edge of the proposed vaned diffuser, see Fig. 1. The area change was made by moving the hub wall, so that 
changes in the development of the shroud wall boundary layer could be clearly seen.

\section{Modified Vaneless Diffuser}

Figure $10 \mathrm{~b}$ shows the meridional flow development for the final converged solution with the modified vaneless diffuser. Now there is no backflow at the diffuser exit, although the shroud boundary layer is close to separation all through the diffuser. All the subsequent flow results presented here are for this modified diffuser.

Flow near the pressure side of the impeller passage is shown in Fig. 10b1. Velocity vectors for flow entering the tip gap are seen all along the shroud. Two other secondary flows also become evident in the second half of the passage; a secondary flow from the hub to the shroud due to curvature of the passage from the axial to the radial direction, and an opposing secondary flow due to the tip leakage flow impinging on the pressure surface. At mid-passage, Fig. $10 \mathrm{~b} 2$ shows the shroud boundary layer thickening and separating in the impeller.

Near the suction side of the impeller, centrifugal and curvature effects combine to give strong secondary flows towards the shroud. This is espectally true in the second half of the passage, where flow angles as high as 30 degrees to the merfdional direction are seen in Fig. $10 \mathrm{~b} 3$.

\section{Therrodynamic Performance}

The thermodynamic performance of the compressor has been evaluated using mass-averaged properties from the 3-D viscous flow calculation. The procedure and nomenclature used follow closely those presented in reference 9. Table 3 presents the thermodynamic state points for the compressor at $1920 \mathrm{RPM}$ and $30 \mathrm{~kg} / \mathrm{s}$.

Four cross-sectional planes are used for these results. Plane 1 is the inlet to the flow domain, $0.188 \mathrm{~m}$ upstream of Plane 1 at the leading edge of the impeller blades. Plane 2 is at the impeller tip and Plane 3 is a plane to be used in preliminary measurements in the vaneless diffuser at a radius ratio $r / r_{2}=1.0667$ ( $r=32$ inches). The states shown 
are static states $(1,1,2,3)$, total states $(t 1, t 1, t 2, t 3)$, relative total states ( $t r 1, t r 1, t r 2, t r 3)$, and rotary stagnation states $\left(* 1, *_{1}\right.$, *2, *3). Also shown is state $u$, reached by isentropic centrifugal pressure rise from state 1 , which is used to describe the impeller performance as a diffuser. The remaining states ( $\left.t 2^{\prime}, t u^{\prime}, 3^{\prime}, t 3^{\prime}\right)$ are used in calculating the isentropic impeller and compressor efficiencies in Table 4. The zero level for entropy is taken at the reference state $(0)$.

The total pressure ratio at the rotor exit is 1.136 compared with the NASA prediction of 1.173 [1]. The static pressure ratio at the rotor exit is 1.087 . 
Table 3. Thermodynamic state points for the compressor at 1920 RPM and $30 \mathrm{~kg} / \mathrm{s}(66 \mathrm{lbm} / \mathrm{s})$.

\begin{tabular}{|c|c|c|c|}
\hline State & $\mathrm{p} / \mathrm{p}_{\mathrm{o}}(1)$ & $\mathrm{T}(\mathrm{K})$ & $\mathrm{s}(\mathrm{J} / \mathrm{kgK})$ \\
\hline 0 & 1.0000 & 288.15 & 0. \\
\hline 1 & $(0.9857)$ & 287.00 & 0.1 \\
\hline$t 1$ & $(0.9996)$ & 288.15 & 0.1 \\
\hline *1 & $(0.9996)$ & 288.15 & 0.1 \\
\hline $\operatorname{tr} 1$ & 1.0263 & 290.33 & 0.1 \\
\hline 1 & $(0.9786)$ & 286.40 & 0.1 \\
\hline$t 1$ & $(1.0004)$ & 288.20 & 0.1 \\
\hline *i & $(0.9998)$ & 288.15 & 0.1 \\
\hline $\mathbf{u}$ & $(1.0936)$ & 295.64 & 0.1 \\
\hline $\operatorname{tri}$ & 1.0296 & 290.59 & 0.1 \\
\hline 2 & $(1.0867)$ & 295.95 & 3.0 \\
\hline$t 2$ & $(1.1362)$ & $299.74^{(2)}$ & 3.0 \\
\hline *2 & $(0.9911)$ & 288.26 & 3.0 \\
\hline $\operatorname{tr} 2$ & 1.1388 & 299.94 & 3.0 \\
\hline$t 2^{\prime}$ & $(1.1362)$ & 298.87 & 0.1 \\
\hline $2 u^{\prime}$ & $(1.0867)$ & $295 \cdot 10$ & 0.1 \\
\hline 3 & $(1.0931)$ & 296.62 & 3.6 \\
\hline$t 3$ & $(1.1348)$ & $299.82^{(2)}$ & 3.6 \\
\hline *3 & $(0.9893)$ & 288.28 & 3.6 \\
\hline $\operatorname{tr} 3$ & 1.1584 & 301.58 & 3.6 \\
\hline $3^{\prime}$ & $(1.0931)$ & 295.61 & 0.1 \\
\hline$t 3^{\prime}$ & $(1.1348)$ & 298.79 & 0.1 \\
\hline
\end{tabular}

(1) Note: the slight inconsistency of these values arises because the
pressures were independently mass averaged. ${ }^{(2)} T_{t 3}$ should equal $T_{t 2}$ for this adiabatic diffuser flow. 
Table 4. Efficiencies and work parameters for this compressor at 1920 $\mathrm{RPM}$ and $30 \mathrm{~kg} / \mathrm{s}(661 \mathrm{bm} / \mathrm{s})$.

$$
\begin{aligned}
& n_{s-s 13}=\frac{T_{3}-T_{1}}{T_{3}-T_{1}} \quad=0.895 \\
& n_{t-t 13}=\frac{T_{t 3}-T_{t 1}}{T_{t 3}-T_{t 1}}=0.912 \\
& n_{\mathrm{p} 13}=\ln \frac{\mathrm{T}_{3}}{\mathrm{~T}_{1}} / \ln \frac{\mathrm{T}_{3}}{\mathrm{~T}_{1}}=0.897 \\
& n_{w}=\frac{T_{2 u^{\prime}}-T_{u}}{T_{2}-T_{u}} \quad=-1.74 \\
& n_{t-t 12}=\frac{T_{t 2}-T_{t i}}{T_{t 2}-T_{t i}} \quad=0.925 \\
& \frac{\Delta H}{U^{2}} \quad=\frac{c_{p}\left(T_{t 2}-T_{t 1}\right)}{\omega^{2} r_{2}^{2}}=0.4960 \\
& \frac{\Delta H^{*}}{U^{2}} \quad=\frac{c_{p}\left(T_{2}^{*}-T_{1}^{*}\right)}{\omega^{2} r_{2}^{2}}=0.0047 \\
& \frac{\Delta H-\Delta H^{*}}{U^{2}} \quad=0.4913 \\
& \begin{array}{ll}
v_{\theta 2} & =0.4914
\end{array}
\end{aligned}
$$




\section{Inpeller and Compressor Bfficiencies}

The total-to-total, static-to-static, and polytropic efficiencies for the compressor between states 1 and 3 are given in Table 4 as 91.2 , 89.5, and 89.7 percent, respectively. These are all significantly below the NASA prediction of 93.4 percent [1]. The total-to-total efficiency of the impeller between states 1 and 2 agrees more closely and is 92.5 percent. These low values can be explained by the very low wheel efficlency, $n_{w}$, which gives a measure of the rotor performance as a diffuser, reducing the relative kinetic energy. Table 4 shows this to be actually negative, at -174 percent (Note the small differences between large numbers here and the large errors possible in this figure. But it does seem to be negative!).

\section{Work Input}

The work input parameter $\Delta H / U^{2}$ is seen in Table 4 to be 0.496 . Most of this work is seen as a change in moment of momentum across the rotor, but some moment of momentum is dissipated at the shroud wall, between the inlet and exit of the impeller, and this appears as an increase in rothalpy $\left(\Delta \mathrm{H}^{*}\right)$ through the impeller. Thus the sum of $\mathrm{V}_{\theta 2} / \omega \mathrm{r}_{2}=0.4914$ and $\Delta \mathrm{H} * / \mathrm{U}^{2}=0.0047$ should equal $\Delta \mathrm{H} / \mathrm{U}^{2}=0.4960$.

The low value of work input is partly due to the high backsweep ( 55 degrees) of the impeller blades. It is partly due also to blockage caused by the thick shroud boundary layer at the lmpeller exit, seen in Fig. 10b2. This causes high relative velocities in the hub half of the impeller passage, and these in turn cause reduced values of absolute velocity. The contours of dimensionless absolute tangential velocity, $V_{\theta 2} / U_{2}$, seen in Fig. 11 in the exit plane of the impeller, therefore show a significant region with values less than 0.5 below passage midheight.

Static Temperature Changes in the Impeller

The static temperature rise in the impeller may be expressed as 


$$
\begin{aligned}
\mathrm{T}_{2}-\mathrm{T}_{1} & =\frac{\mathrm{W}_{1}^{2}-\mathrm{W}_{2}^{2}}{2 c_{\mathrm{p}}}+\frac{\mathrm{U}_{2}^{2}-\mathrm{U}_{1}^{2}}{2 c_{\mathrm{p}}}+\mathrm{T}_{2}^{*}-\mathrm{T}_{1}^{*} \\
& =(4.19-3.99)+(11.68-2.44)+(288.26-288.15) \\
& =0.20+9.24+0.11 \\
& =9.55 \mathrm{~K}
\end{aligned}
$$

The centrifugal effects clearly dominate in this rotor and there is a surprisingly small contribution, only 2 percent, from the reduction of the relative kinetic energy. This is also seen in Fig. 12 where the development of the mass-averaged values of relative kinetic energy and kinetic energy associated with the local wheel speed may be followed. The relative kinetic energy falls linearly in the first half of the impeller passage but then it increases, due in part to excessive shroud boundary layer blockage and excessive backsweep.

\section{Total Temperature Changes in the Impeller}

The total temperature rise in the impeller may be written

$$
\begin{aligned}
T_{t 2}-T_{t 1} & =\frac{W_{1}^{2}-W_{2}^{2}}{2 c_{p}}+\frac{U_{2}^{2}-U_{1}^{2}}{2 c_{p}}+T_{2}^{*}-T_{1}^{*}+\frac{v_{2}^{2}-v_{i}^{2}}{2 c_{p}} \\
& =(4.19-3.99)+(11.68-2.44)+(288.26-288.15)+(3.79-1.80) \\
& =0.20+9.24+0.11+1.99 \\
& =11.54 \mathrm{~K}
\end{aligned}
$$

Again the impeller performance is seen to be dominated by centrifugal effects. The absolute kinetic energy at the exit of the impeller is small, and there appears to be a limited potential for contributions from the diffuser downstream of the impeller. Said another way, the reaction of the impeller is large: in fact, 


$$
\text { Reaction }=\frac{T_{2}-T_{1}}{T_{t 2}-T_{t i}}=\frac{9.55}{11.54}=0.828 .
$$

\section{3-D Pressure Distribution}

The three-dimensional distribution of reduced static pressure throughout the compressor flow domain is shown in Figs. 13, 14 and 15 as plots and contours of isentropic relative Mach number.

\section{Trpeller Blade Loading}

The calculated blade loading for hub and tip sections of the impe1ler is shown in Fig. 13. Also shown, repeated from Fig. 2, are the NASA predictions made using a 3-D Euler code [3]. The present calculation conflrms the uniform blade loading predicted by the Euler code, and the loading distribution at the hub agrees reasonably well. But there is a significant difference at the blade tip section. The 3-D flow calculation with the pressure-correction method shows diffusion only in the first half of the impeller passage, followed by a slight reacceleration in the second half. As discussed above, this is partly due to shroud boundary layer blockage in the present calculation. Unlike the calculation with the Euler code, the present results show a quite uniform rotor exit static pressure distribution.

\section{Blade Surfaces}

Figure 14 shows the surface Mach numbers on the pressure and suction sides of the impeller blades, together with results on grid surfaces extrapolated upstream and downstream. Figure $14 a$ shows the minimum Mach number in the hub-pressure-side corner region to be about 0.17 , and Figure 14b shows a maximum surface Mach number of 0.35 on the suction surface in the inducer. Diffusion in the impeller is seen to be limited mostly to the shroud half of the passage and there it is complete by mid-way along the impeller. The static pressure is quite uniform from hub to shroud near the impeller exit and in the diffuser downs tream. 
Hub Wall and Blade TIp Region

Isentroplc Mach numbers on blade-to-blade planes are shown in Fig. 15. At the hub, the Mach number varies from about 0.2 in the Inducer to about 0.27 at the impeller exit, as seen in Fig. 15a. Near the shroud wall two sections are shown, one at about 80 percent of blade height in Fig. $15 b$ and one at the height of the blade tip in Fig. 15c. The differences between the two planes are quite small in this calculation, of the order of 0.025 in Mach number in the inducer. Overall the Mach numbers change very little from about 0.31 in the inducer to about 0.28 at the impeller exit.

\section{Shroud Static Pressure Distribution}

At the shroud wall, the static pressure distribution, normalized with the inlet total pressure, $p_{0}$, is as shown in Fig. 16. The pressure ratio varies from a minimum of about 0.96 on the suction side in the Inducer to an average value of 1.087 at the impeller exit. This steady pressure rise is mostly due to centrifugal effects.

\section{3-D Flow Development}

Blade-to-Blade Planes

Throughflow velocity vectors projected onto blade-to-blade planes are presented in Fig. 17. A plane near the hub, at a fraction $C=0.003$ of passage height, is shown in Fig. 17a, while Fig. 17b shows the results at 80 percent of passage height. The flow appears quite uniform at the hub with little evidence of variations due to blade loading or impeller diffusion. At the 80 percent height, there appears to be some diffusion (slowing of the relative velocity) and some evidence of blade loading in the latter half of the passage, but these effects may also be due to a thickening of the shroud boundary layer. On both of these planes secondary flows appear small.

Much stronger secondary flows are found at the mid-height of the blade tip gap, as seen in Fig. 17c. The convection of tip leakage flow across the shroud wall towards the pressure side can be seen in the 
inducer; the leakage flow appears to reach the next tip gap about 40 percent along the blade; and after that, there is backflow in the meridional direction in the shroud wall boundary layer. The tip leakage flow crosses the blade tip obliquely in the inducer and there is a strong component in the throughflow direction in the tip gap. In the latter half of the impeller, however, the tip leakage becomes more normal to the blade, contributing significantly to the backflow.

\section{Cross-Sectional Planes}

The non-orthogonal representation of secondary flow velocity vectors, introduced in reference 5, is used in Fig. 18 to aid in visualizing the secondary flows. The results are shown on four planes, at 10 , 40, 74 and 100 percent of meridional distance, A, through the impeller. Contours of primary flow velocity, normalized by the impeller tip speed $\mathrm{U}_{2}=153 \mathrm{~m} / \mathrm{s}$, are also shown in an attempt to give a complete picture of the three-dimensional flow development.

The strongest secondary flows are clearly associated with the tip leakage flow and its subsequent convection and mixing in the passage. As seen in Fig. $17 \mathrm{c}$, the tip leakage fluid convects across the shroud wall; in Fig. $18 \mathrm{a}$ at 10 percent meridional distance it is just emerging from the tip gap; in Fig. $18 \mathrm{~b}$, at $A=0.40$, it has fust reached the pressure side and is flowing into the next tip gap. Some mixing and recirculation of the tip leakage fluid is evident in Fig. $18 \mathrm{~b}$ at the edge of the shroud boundary layer at about 80 percent of passage height.

In the second half of the passage, the tip leakage fluid impinges on the pressure surface of the next blade, causing secondary flow towards the hub. This was also seen in the meridional view of the velocity vectors near the pressure surface in Fig. $10 \mathrm{bl}$. By $A=0.74$, the tip leakage flow and recirculation are well established features of the whole flow, as seen in Fig. 18c. Their influence remains at the Impeller exit, seen in Fig. 18d; and they explain the distributions of $\mathrm{V}_{\theta 2} / \mathrm{U}_{2}$ seen in the shroud half of the Impeller passage in Fig. 11. The contours of 0.6 and 0.7 are caused by recirculating tip leakage fluid. 
of the other secondary flows evident in Fig. 18, the migration of the suction surface boundary layer towards the shroud wall is the most extensive. This was noticed particularly in Fig. $10 \mathrm{~b} 3$.

\section{Detail of Flow through Tip Gap}

The calculated secondary velocity vectors and primary velocity contours for flow over the blade tip are shown in Fig. 19. Figure 19a shows the tip gap flow just starting in the inducer, while Fig. 19b shows a more developed leakage flow pattern with repeat leakage and entrainment of suction surface boundary layer flow.

\section{Cumulative Flow through Tip Gap}

The cumulative fraction of the passage mass flow that passed through the tip gap bullds up quite linearly, as seen in Fig. 20. This is due to the uniform height of the tip gap and the uniform blade loading. The overall leakage is 18 percent, although most of the tip leakage fluid appears to have passed over more than one blade tip.

\section{Los8 Distributions}

Distributions of entropy on cross-sectional planes through the impeller and the vaneless diffuser are presented in Fig. 21. The contour interval is $1.0 \mathrm{~J} / \mathrm{kgK}$.

In the inducer the flow is modelled as having turbulent boundary layers and these are of relatively uniform thickness. The suction surface boundary layer thickens by mid-passage and then convects towards the shroud, as seen in Figs. 21a, b and $c$.

The accumulation of loss in the shroud boundary layer is the main feature of the impeller loss development. Initially the shroud boundary layer is thin (see FIg. 21a), but then it receives an input of tip leakage losses. The losses spread rapidly away from the shroud wall in the second half of the impeller passage (see Fig 21c and 21d). By the impeller exit, they cover most of the shroud/pressure-side quadrant of the passage. The rest of the flow is mostly low loss, potential flow, apart from a re-thickened suction side boundary layer. 
The mixing downstream of the impeller is not very rapid. A comparison of Figs. $21 \mathrm{~d}$ and $21 \mathrm{f}$ shows some thickening of the hub and shroud boundary layers and a spreading of the blade wake. But the high loss region from the shroud/pressure-side quadrant is relatively unchanged by $r / r_{2}=1.08$.

\section{DISCUSSION OF RESULTS}

\section{Tip Leakage Flow Mixing}

The NASA low-speed centrifugal compressor in its initial configuration has a tip clearance gap in the inducer much larger than the two impellers discussed in reference 9. The NASA rotor has a clearance gap equal to 2.6 percent of the inlet blade helght, whereas in the DFVLR and Rolls-Royce Impellers the gaps were 0.5 and 1.0 percent, respectively. The impeller has been set up for large tip leakage flows and that is what is found in the present calculation. The cumulative flow predicted to pass through the tip gap is 18 percent compared with 12 percent for the Rolls-Royce impeller.

In passing over the blade tip, the fluid experiences increases in loss and turbulence kinetic energy. And, in the second half of the impeller passage, some of the leakage fluid passes through more than one tip gap. So there is enhanced turbulence in the boundary layer near the shroud wall. Then, too, some of this leakage fluid recirculates back towards the suction side after impinging on the pressure surface. So the outer layers of the shroud boundary layer may also have high turbulence.

offsetting this, for example, could be the curvature of the shroud wall which could interact with the boundary and mixing layers to suppress turbulence. An estimate of the ratios, $\delta / R$, of the shroud shear layer thicknesses to the radius of curvature of the streamlines suggests that these could be of the order of 0.1. Adams and Johnston [10] suggest that the Prandtl mixing length, $L$, could then be $0.025 \delta$ instead of $0.08 \delta$ as modelled in the present calculations. The turbulent viscosity which depends on $\mathrm{L}^{2}$ would then be a factor of ten smaller than predicted here. With the dominant influence of tip leakage flow and 
mixing on the 3-D impeller flow development found here, it would seem useful to study the effect of such mechanisms on the turbulent mixing.

\section{Jet-Wake Mixing}

Jet-wake mixing in the vaneless diffuser at the exit of the NASA impeller appears to involve the jet in the hub half of the passage slowly mixing with high loss fluid in the shroud half. Superposed on this is a tangential spreading of the blade wakes which also appears to be quite slow in the present calculation.

\section{Backflow Along Inpeller Shroud Wall}

Reverse flow in the meridional direction along the shroud wall is found from 33 to 114 percent of meridional distance in the impeller. It is seen in Fig. $17 \mathrm{C}$ as vectors with a negative meridional component, and it is seen in Figs. 18b, 18c, 18d and $19 \mathrm{~b}$ bounded by contours (thick solid lines) of zero primary velocity. The picture in Fig. 18c at 74 percent meridional distance is quite close to the section at 77 percent where the maximum backflow is about 2.5 percent of the throughflow. Here the thickness of the reverse flow region reaches a maximum of about $1 \mathrm{~cm}$. At this same impeller location, Fig. 19b shows that all the flow in the tip clearance gap has a component in the reverse direction.

Backflow was also seen in the calculations by Moore and Moore [5] for the NACA 48-Inch Wheel. And 1ts possible significance as a precursor of impeller stall has been discussed recently by Chen et al. [11]. Apparently such reverse flows may have a detalled vortex filament structure.

\section{Comparison with HASA Flow and Performance Predictions}

Table 5 compares the performance predictions which have been made in this report with those made in the NASA design study [1]. Since the NASA study was for the compressor with its wedge-type vaned diffuser, comparisons are made for the rotor performance only. 
The present calculation predicts a poorer performance for the current impeller configuration. The reasons for this different prediction are probably associated with the different impeller exit static pressure distributions and the different models of shroud boundary layer development and tip leakage flow mixing. The meridional flow and Euler codes used by NASA both predict higher impeller diffusion and correspondingly better compressor performance.

Table 5. Comparison of Rotor Performance Predictions at 1920 RPM and 30 $\mathrm{kg} / \mathrm{s}(661 \mathrm{bm} / \mathrm{s})$.

$\begin{array}{lll} & \text { NASA [1] } & \begin{array}{l}\text { Present } \\ \text { Calculation }\end{array} \\ \frac{\mathrm{p}_{t 2}}{\mathrm{P}_{\mathrm{O}}}(1) & 1.173 & 1.136 \\ \eta_{t-t 12} & 0.934 & 0.925 \\ \frac{\Delta \mathrm{H}}{\mathrm{U}^{2}} & 0.607 & 0.496 \\ \mathrm{M}_{\text {re11,tip }} & 0.31 & 0.31 \\ \mathrm{M}_{\text {re12 }} & 0.20 & 0.260 \\ \mathrm{M}_{\text {abs2 }} & 0.287 & 0.253 \\ \text { Reaction } & 0.763 & 0.828\end{array}$

(1) Plane 1 is at the impeller inlet; Plane 2 is at the impeller exit. 


\section{CONCLUSIONS}

A three-dimensional turbulent flow calculation has been made for the NASA Low-Speed Centrifugal Compressor Impeller. The calculation was performed with the Moore Elliptic Flow Program, a 3-D pressure-correction solution procedure for discretized forms of the Navier-Stokes equations. A Prandtl mixing-length turbulence model was used.

The calculation was made at the compressor design conditions of $1920 \mathrm{RPM}$ and $30 \mathrm{~kg} / \mathrm{s}$, at $1 \mathrm{~atm}$ and $288.15 \mathrm{~K}$. The tip clearance gap was a uniform 0.225 inches $(5.7 \mathrm{~mm})$. The constant-height vaneless diffuser was modified to have a constant area starting at a radius $\mathrm{ratio} r / r_{2}=$ 1.08 .

The predicted performance is significantly worse than that predicted in the NASA study. The work done factor $\Delta \mathrm{H} / \mathrm{U}^{2}$ is 0.496 compared with 0.607 . The total pressure ratio is also therefore lower, 1.136 compared with 1.173. The total-to-total impeller efficiencies agree reasonably well, 92.5 percent in the present calculation and 93.4 percent predicted by NASA.

The thermodynamic performance of the impeller is dominated by centrifugal effects. The efficiency of the impeller as a diffuser, reducing the relative kinetic energy, 1.e. the wheel efficiency, is negative. The actual value calculated is -174 percent. Previous calculations made by the authors for other centrifugal compressor impellers have given values of about +60 percent. But those impellers were run with significantly smaller tip clearances in the inducer, 0.5 and 1.0 percent of blade height, compared with 2.6 percent, here.

The tip clearance varied through the impeller from 2.6 percent to 4.0 percent. Combined with the relatively uniform blade loading, the resultant tip leakage was 18 percent of the passage throughflow. The Impeller flow development is therefore strongly influenced by the convection and mixing of tip leakage fluid.

Tip leakage flow passes across the shroud wall; some of the flow Impinges on the pressure surface of the next blade, while the rest passes through the next tip gap. A region of high losses develops in 
the shroud/pressure-side quadrant of the impeller passage. This "wake" mixes relatively slowly with the "jet" in the hub half as the flow passes through the vaneless diffuser.

The boundary layer on the shroud wall exhibits backflow in the meridional direction over the last two-thirds of the impeller length. The reverse flow is a maximum of 2.5 percent of the throughflow about three-quarters of the way along the impeller. Here, the maximum thickness of the backflow region was about $1 \mathrm{~cm}$ in the present calculation.

The 1mpeller with its initial unfform tip clearance setting will offer the opportunity to study a three-dimensional impeller flow dominated by tip leakage. Tip leakage mixing layers, similar to those predicted previously by the authors in a helicopter engine impeller, can be studied. In the NASA low-speed centrifugal compressor impeller these layers will be of the order of 1-2 inches thick. This should be an ample size for detailed turbulence measurements and flow definition.

\section{RECOMENDATIONS FOR SECOND PHASE TESTING}

1. The geometry of the vaneless diffuser should be modified to prevent significant reverse flows on the shroud wall. In the present 3-D flow calculation, a constant area vaneless section, starting at a radius ratio $r / r_{2}=1.08$ (see $F i g$. $5 b$ ), accomplished this.

2. Tip leakage flow mixing should be studied in the impeller. Particular attention should be given to the shroud wall boundary layer and to loss accumulations in the shroud/pressure-side quadrant. Turbulence modification, for example due to curvature of the streamlines, may significantly affect turbulent mixing in these regions.

\section{RECORARNDATIONS FOR THIRD PHASE TESTING}

1. The tip clearance gap in the inducer region of the impeller should be reduced to $1 \%$ of blade height or less. The reduction should be extended along the impeller until a wheel efficlency (see earlier discussion) of at least 0.5 is achieved. 


\section{REFERENCES}

1. Wood, J. R., Adam, P. W., and Buggele, A. E., "NASA Low-Speed Centrifugal Compressor for Fundamental Research," NASA TM-83398, June 1983.

2. Katsanis, T., and McNally, W. D., "Revised Fortran Program for Calculating Velocities and Streamlines on the Hub-Shroud Midchannel Stream Surface of an Axial-, Radial-, or Mixed-Flow Turbomachine or Annular Duct: Part I - User's Manual," NASA TN D-8430, March 1977.

3. Denton, J. D., "An Improved Time Marching Method for Turbomachinery Flow Calculation," Trans. ASME, Journal of Engineering for Power, Vo1. 105, No. 3, 1983, pp. 514-524.

4. Moore, J., and Moore, J. G., "3-D Viscous Flow Calculations at Design and Off-Design Conditions for the NACA 48-Inch Radial Inlet Centrifugal Impeller," Proceedings of the Eighth International Symposium on Air Breathing Engines, pp. 139-148, Cincinnati, Ohio, AIAA Paper ISABE 87-7008.

5. Moore, J., and Moore, J. G., "Secondary Flow, Separation and Losses in the NACA 48-Inch Centrifugal Impeller at Design and Off-Design Conditions," ASME Paper No. 88-GT-101.

6. Moore, J. G., "Calculation of 3-D Flow Without Numerical Mixing," AGARD Lecture Series No. 140 on "3-D Computational Techniques Applied to Internal Flows in Propulsion Systems," June 1985.

7. Moore J. G., "An Elliptic Calculation Procedure for 3-D Viscous Flow," AGARD Lecture Series No. 140, loc. cit.

8. Moore, J., Moore, J. G., Henry, G. S., and Chaudhry, U., "Flow and Heat Transfer in Turbine Tip Gaps," ASME Paper No. 88-GT-188.

9. Moore, J., "Performance Evaluation of Flow in Turbomachinery Blade Rows," AGARD Lecture Series No. 140, 1oc. cit.

10. Adams, E. W., and Johnston, J. P., "A Mixing-Length Model for the Prediction of Convex Curvature Effects on Turbulent Boundary Layers," Trans. ASME, Journal of Engineering for Gas Turbines and Power, Vol. 106, No. 1, January 1984, pp. 142-148.

11. Chen, Y. N., Haupt, U., and Rautenberg, M., "The Vortex-Filament Nature of the Reverse Flow on the Verge of Rotating Stall," ASME Paper No. 88-GT-120. 


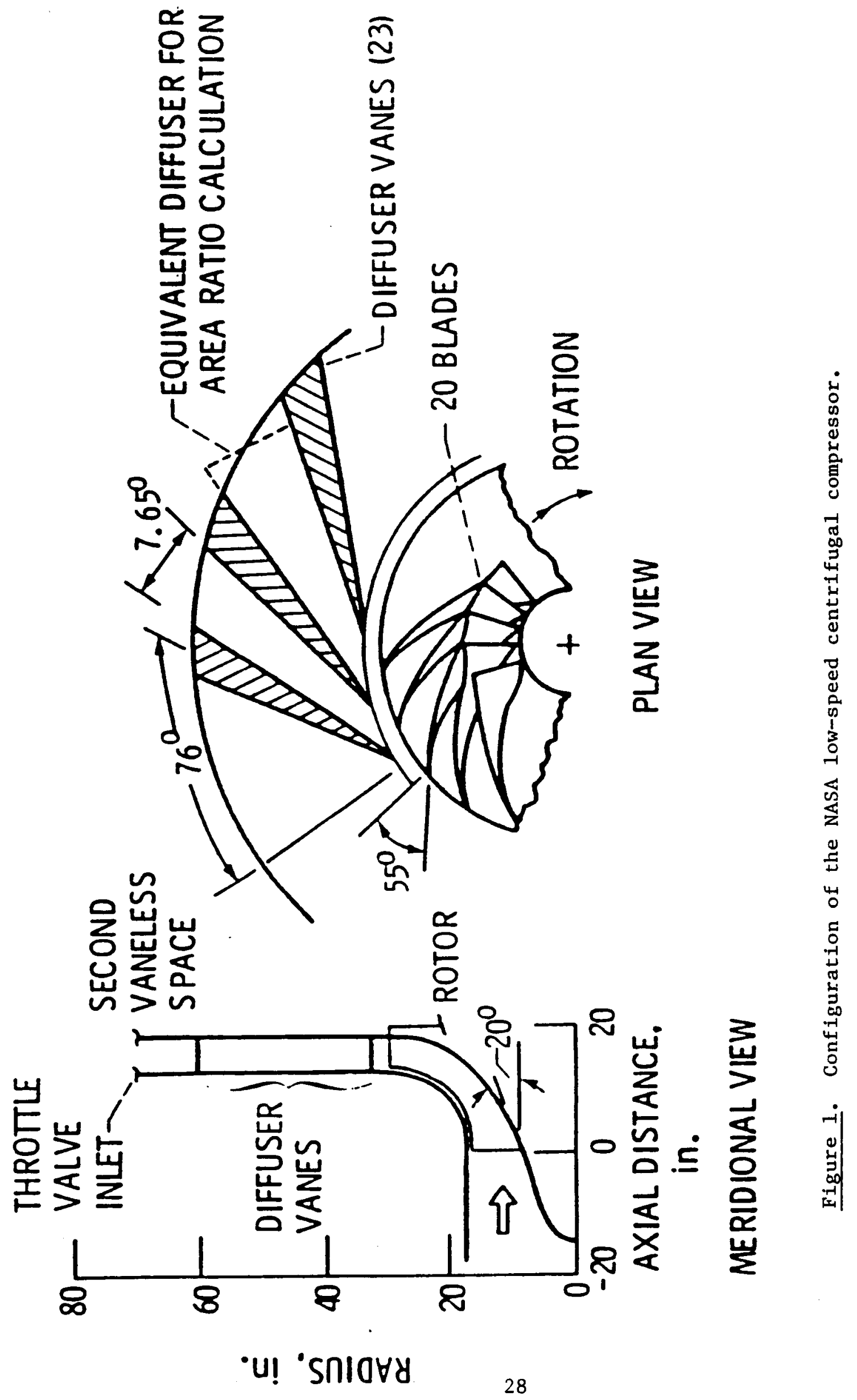



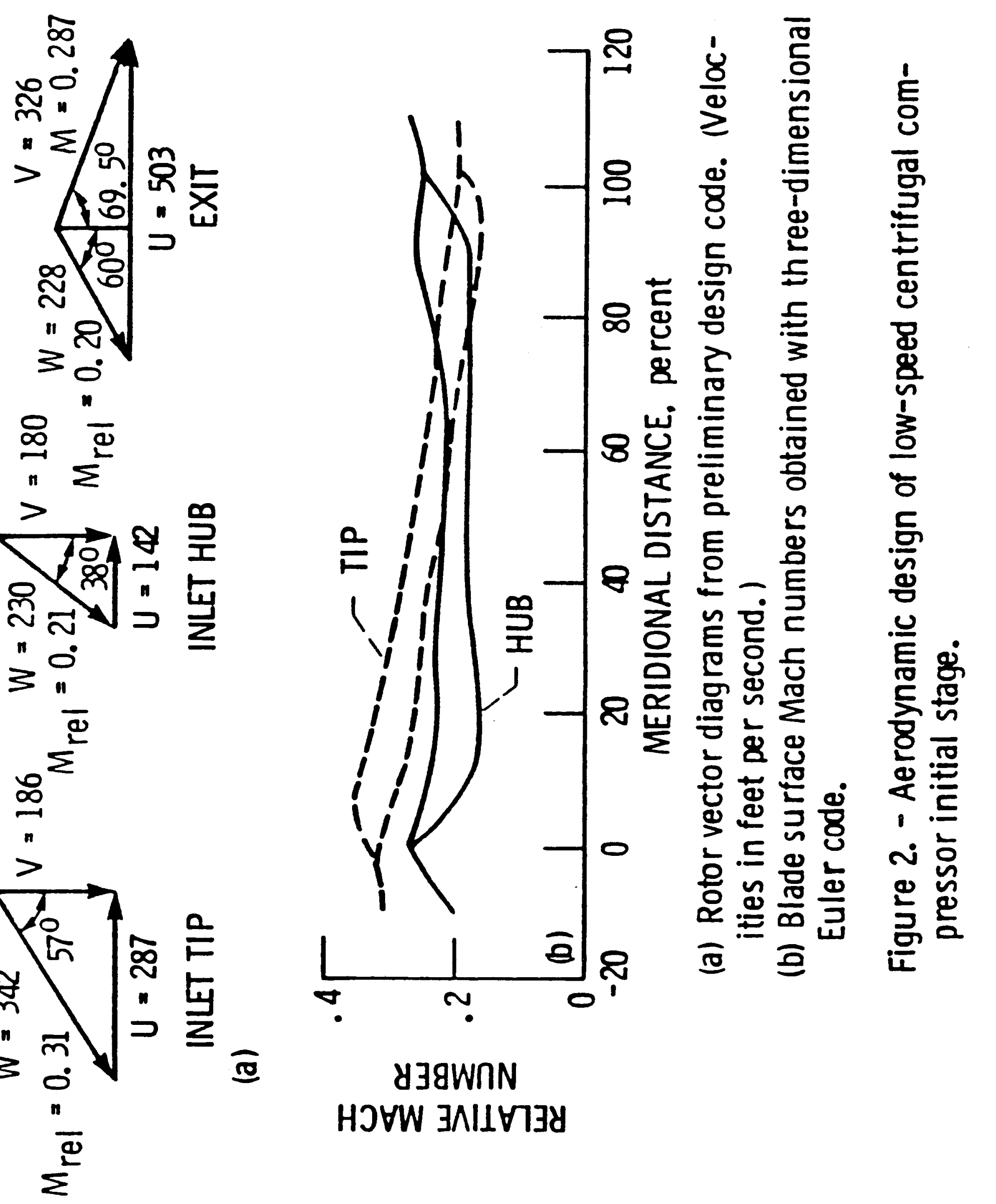


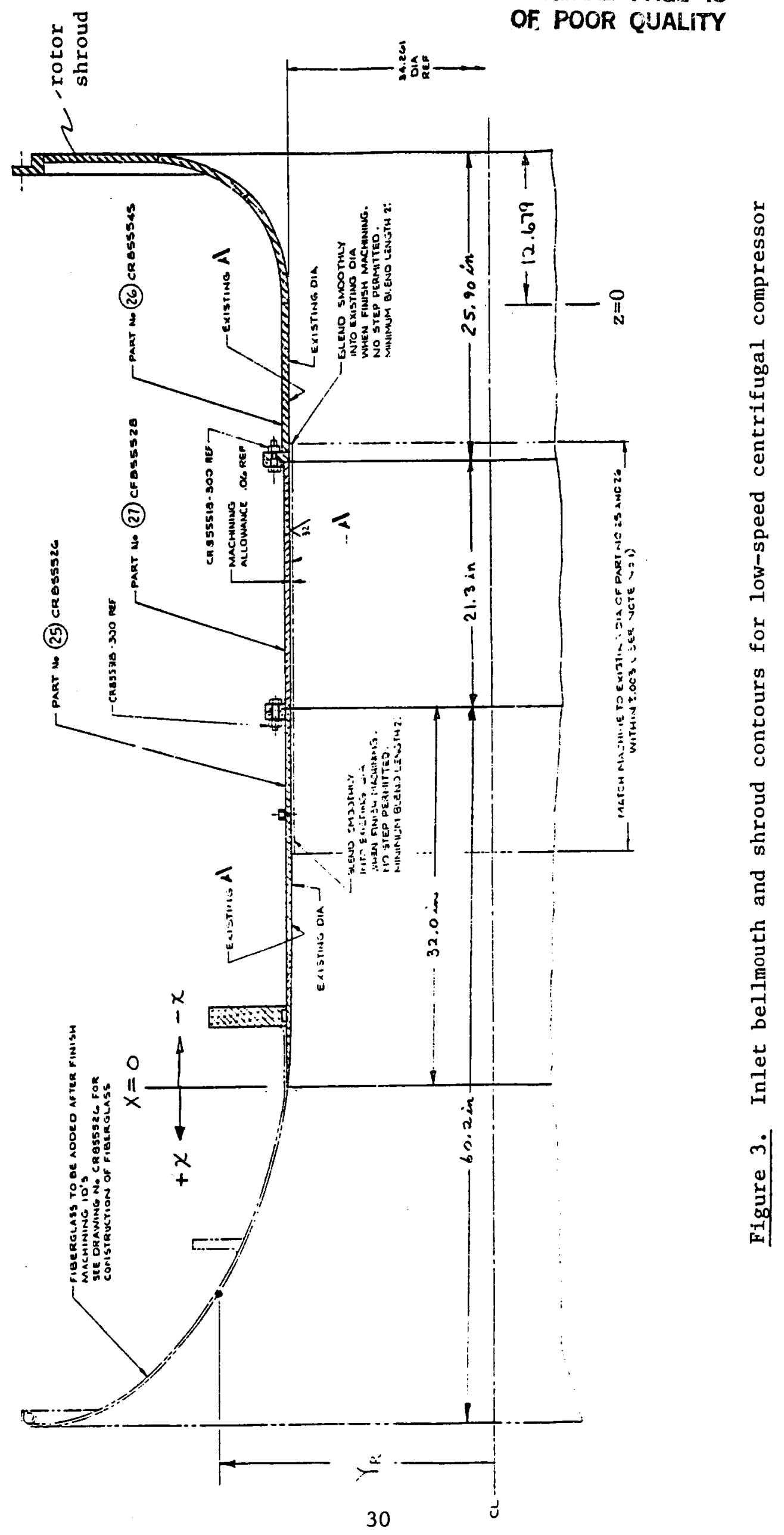


Fig. 5a. Constant-height vaneless diffuser.

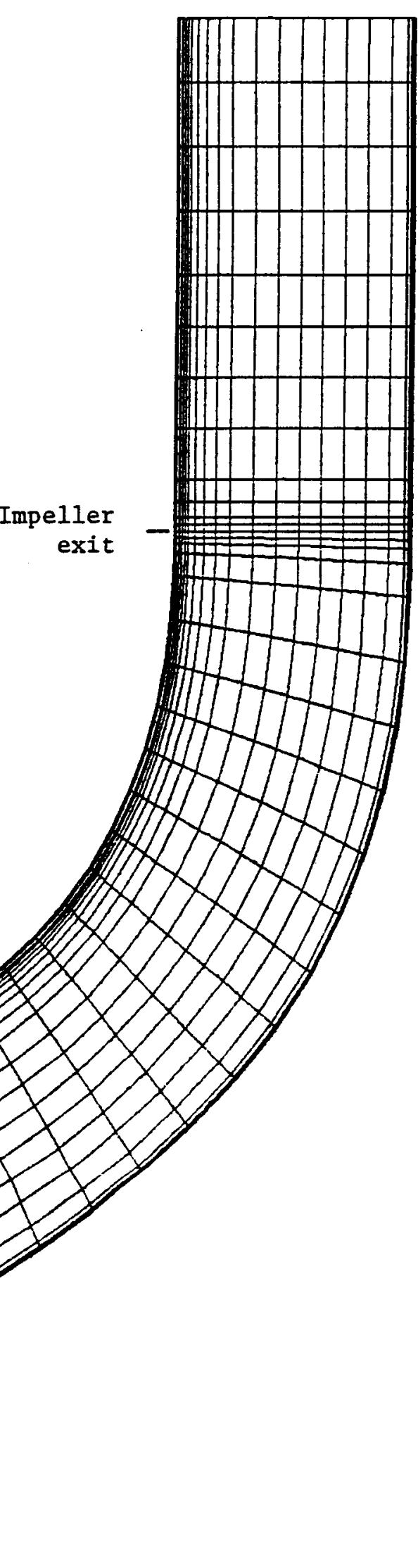

Figure 5. Meridional view of grids for 3-D flow calculations. 
Fig. 5b. Modified vaneless diffuser with constant area from $r / r_{2}=1.08$.

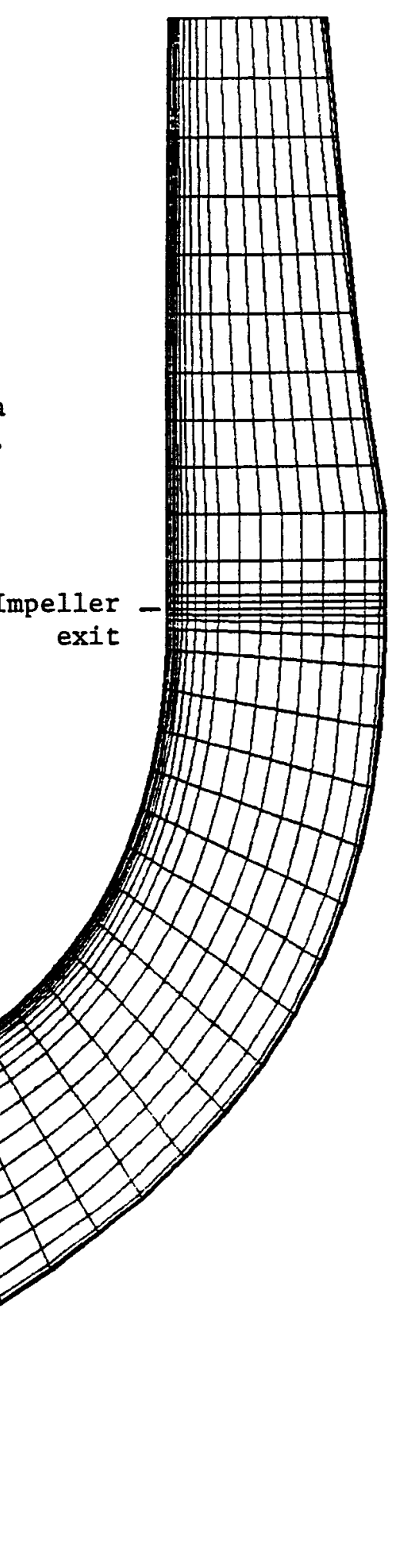




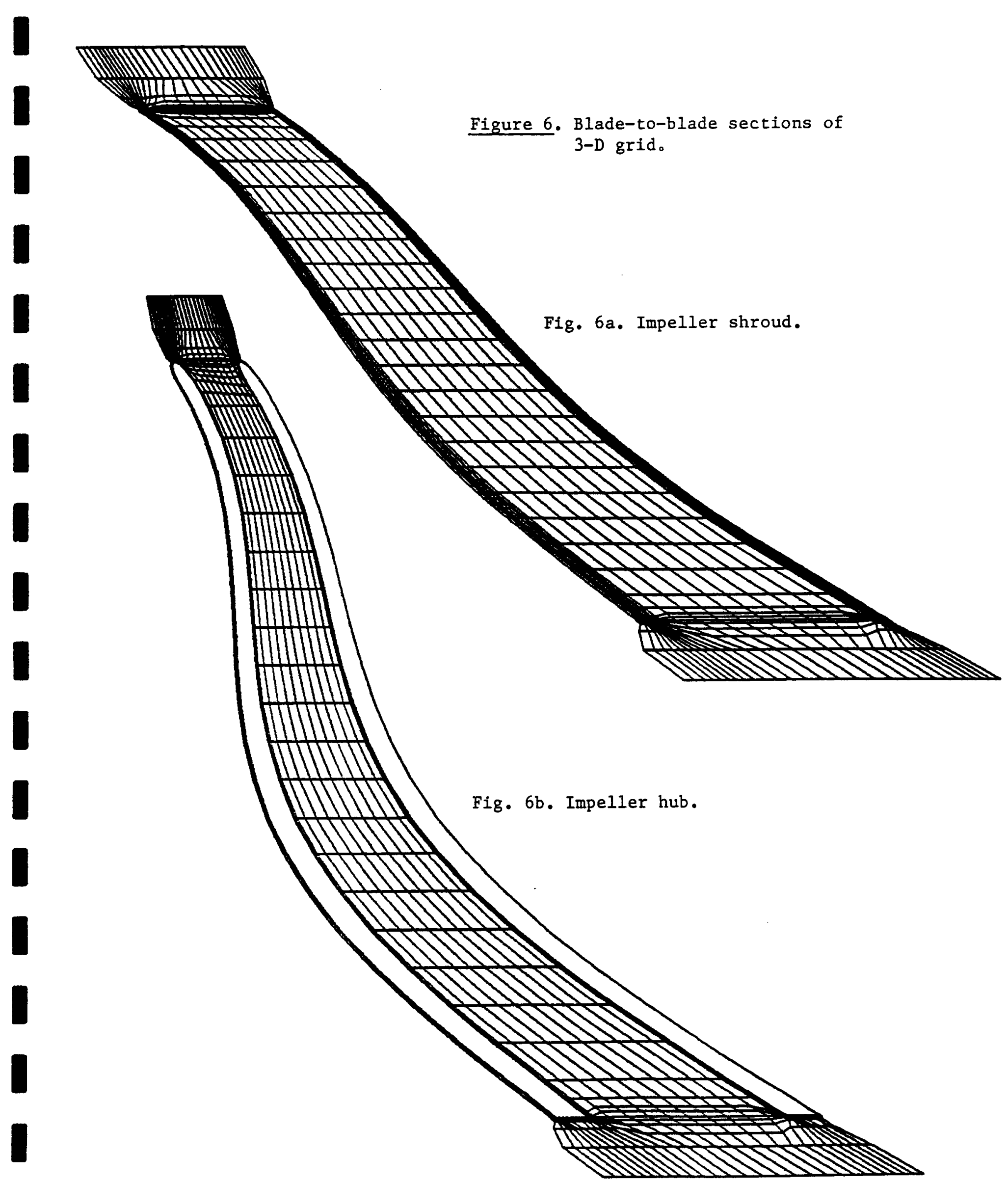



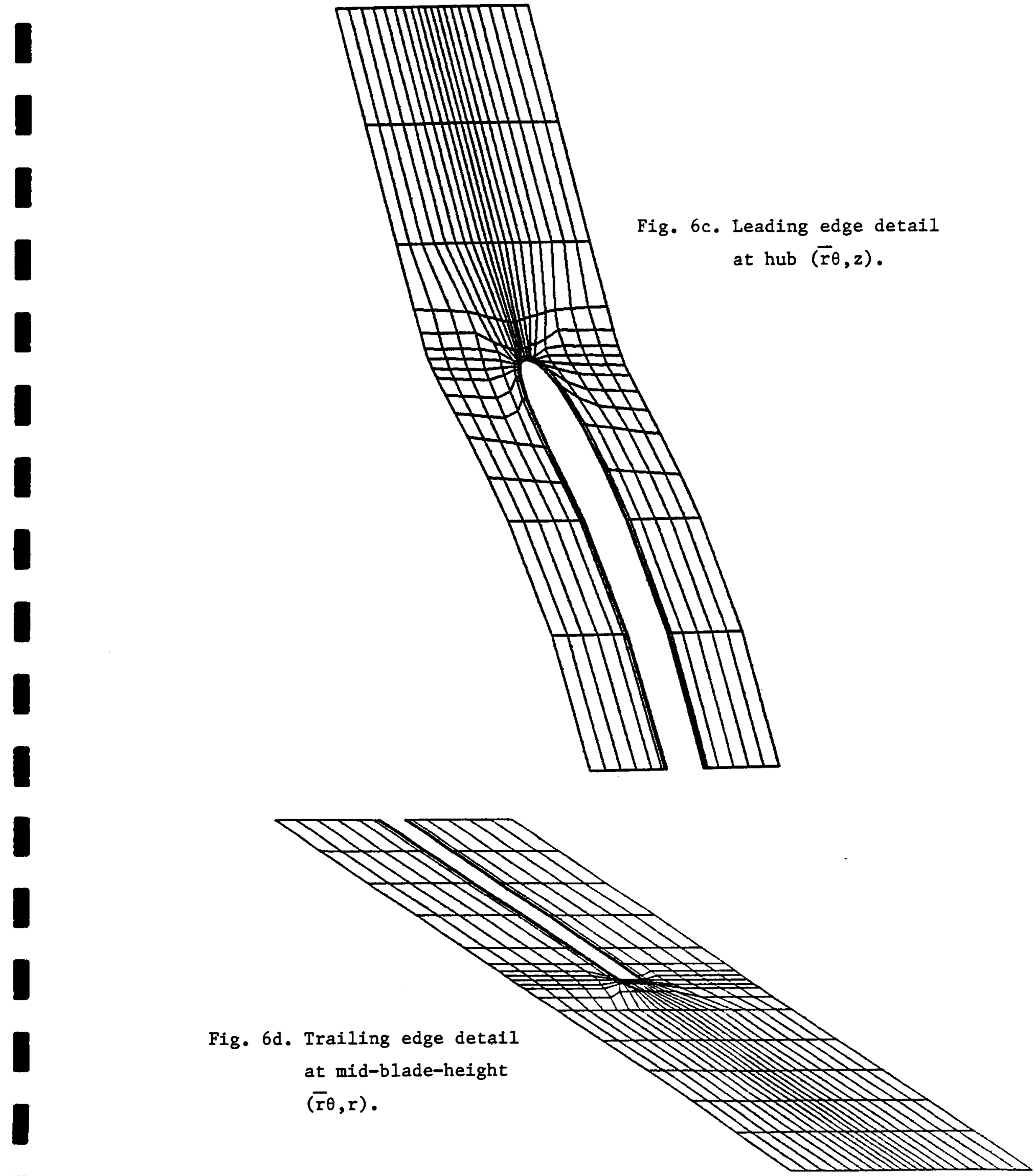
Fig. 7a. Impeller exit $(\overline{\mathrm{r}} \theta, z)$.

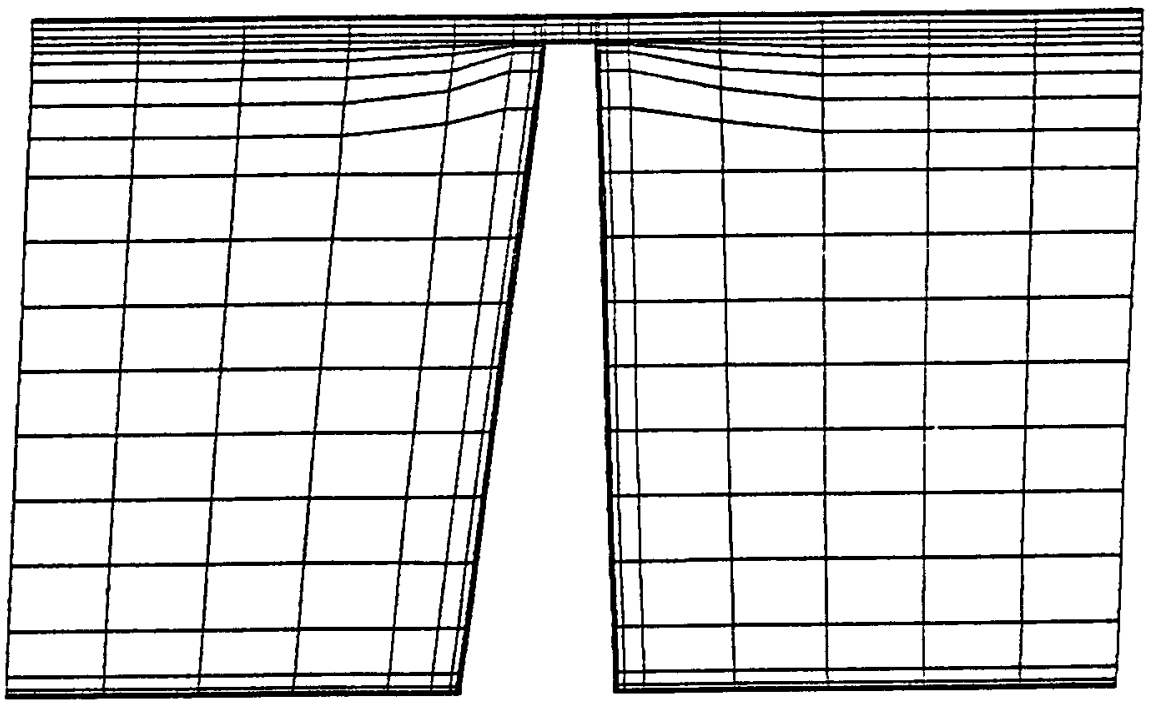

Fig. 7b. Near impeller inlet, at $4.1 \%$ meridional distance from leading edge. $\overline{(r} \theta, r)$.

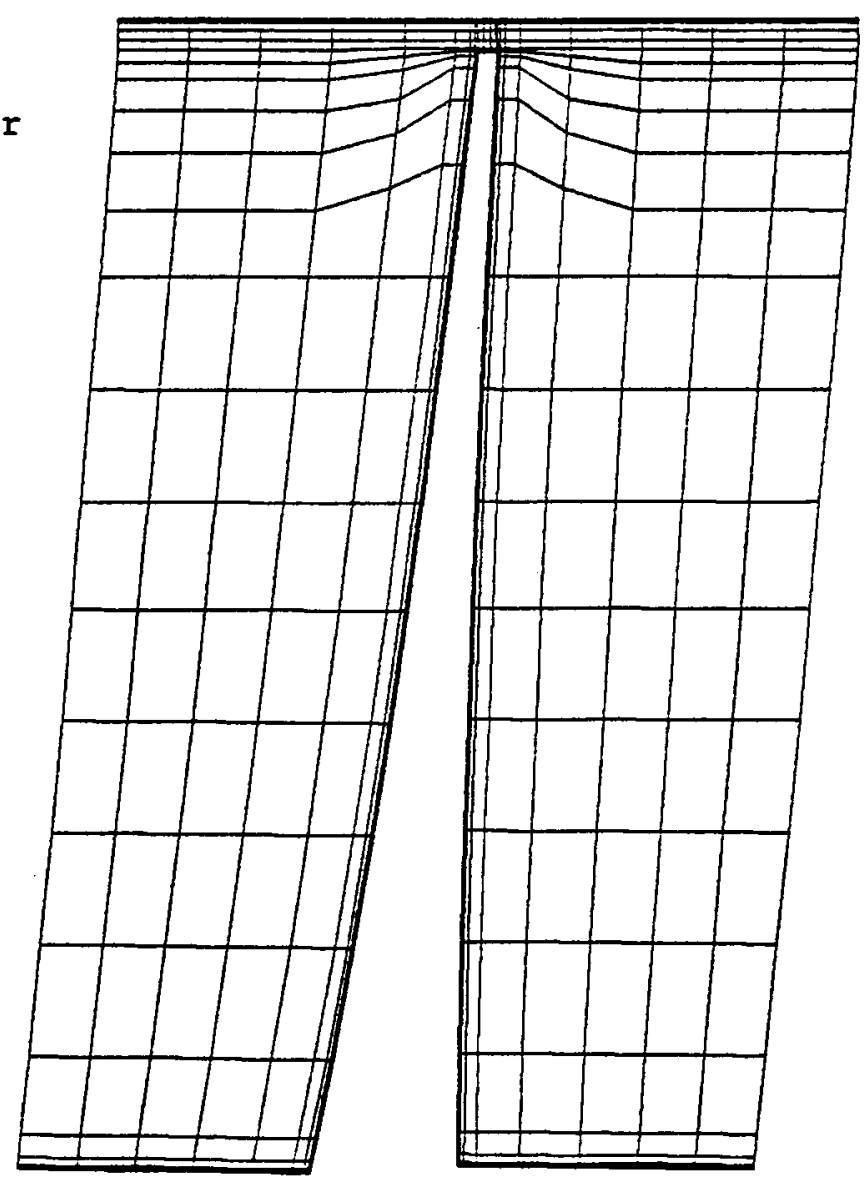




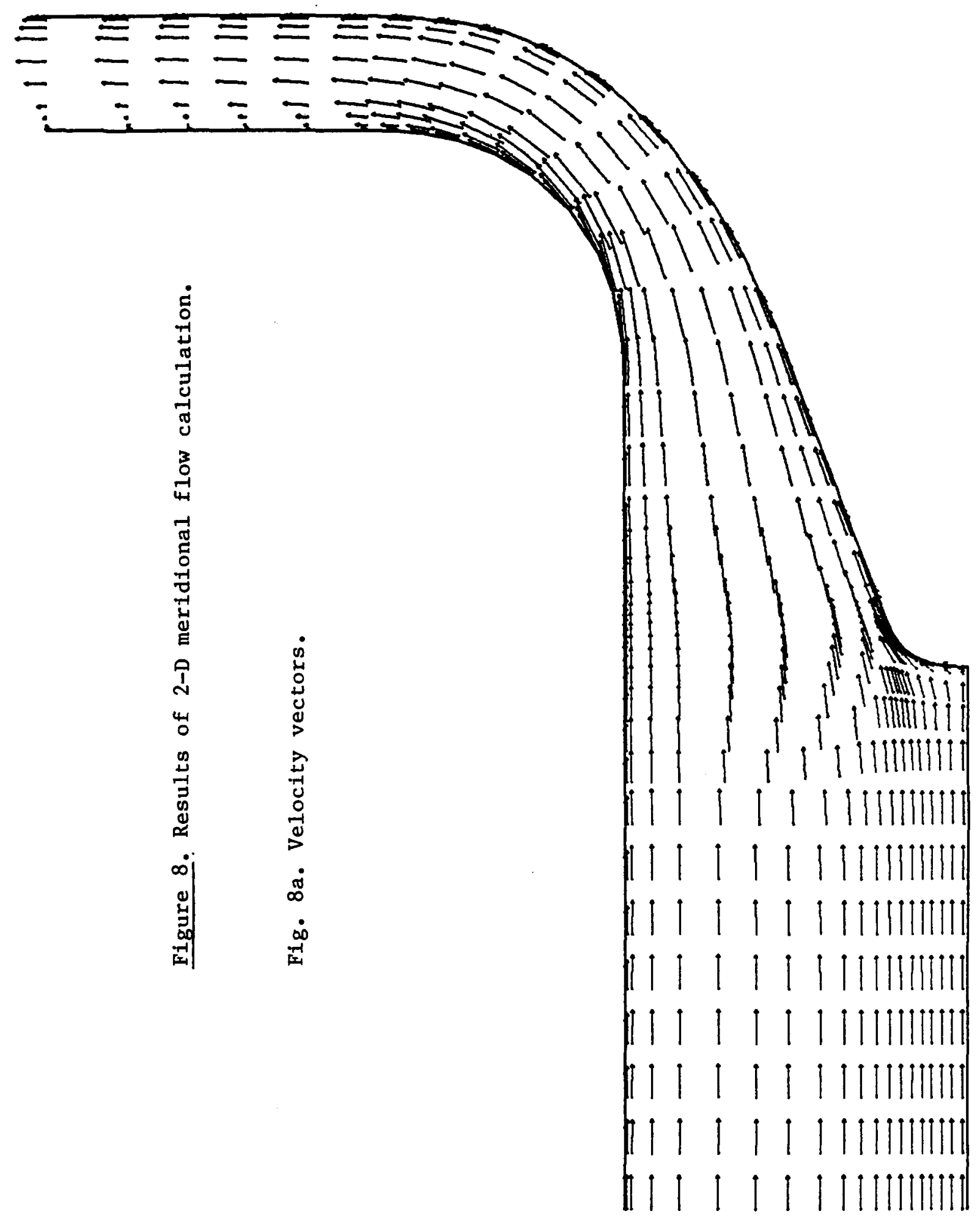




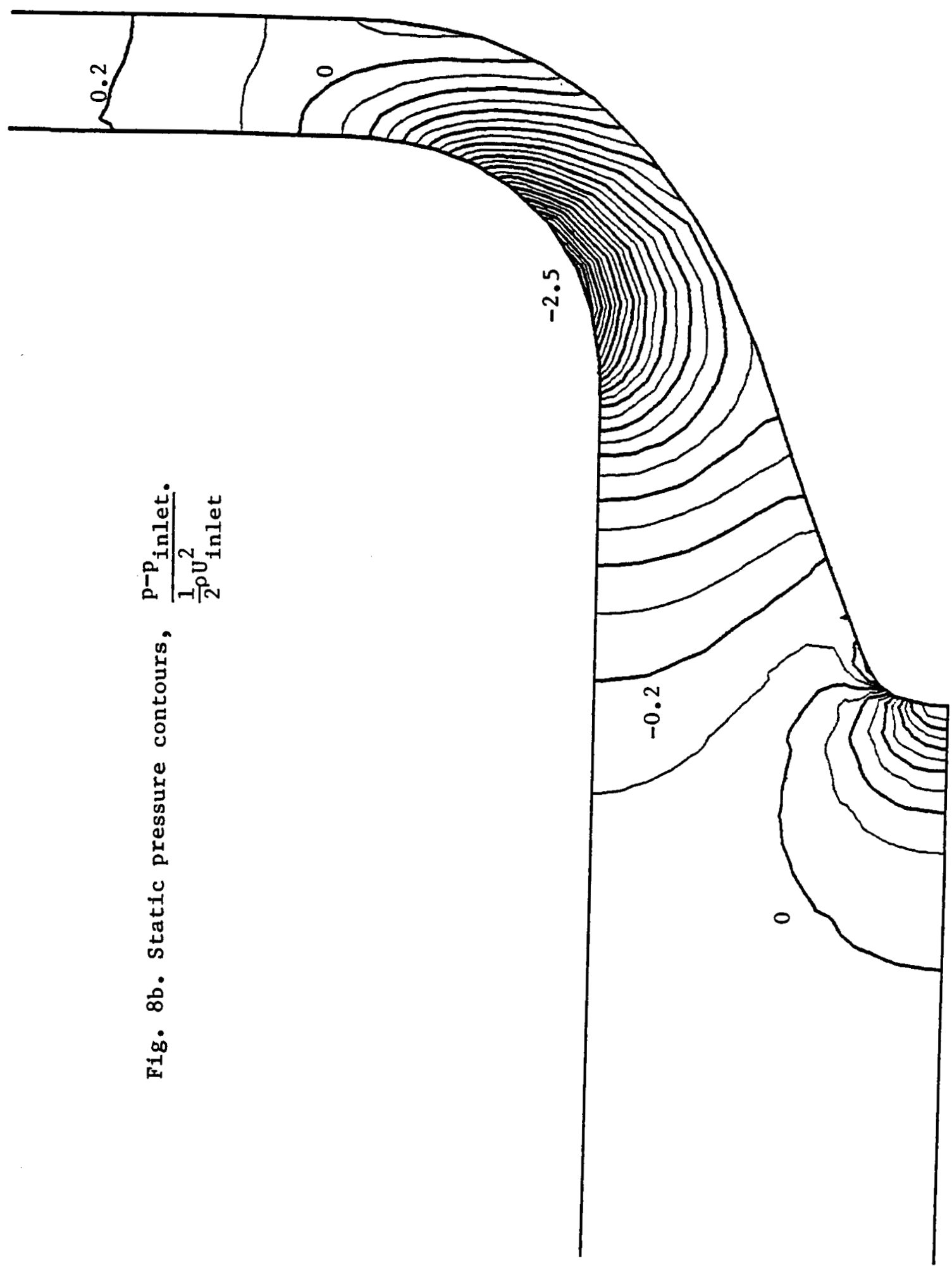



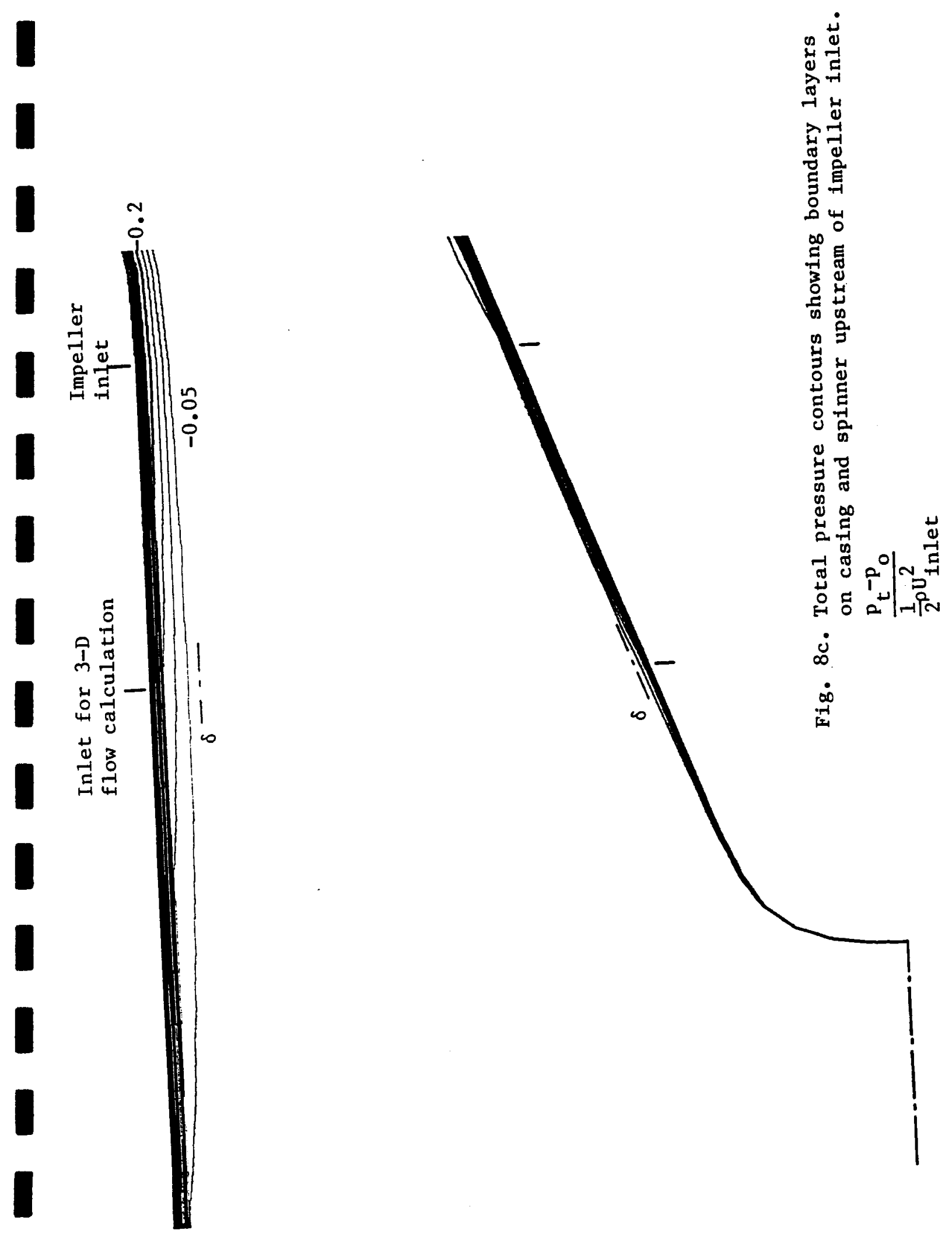


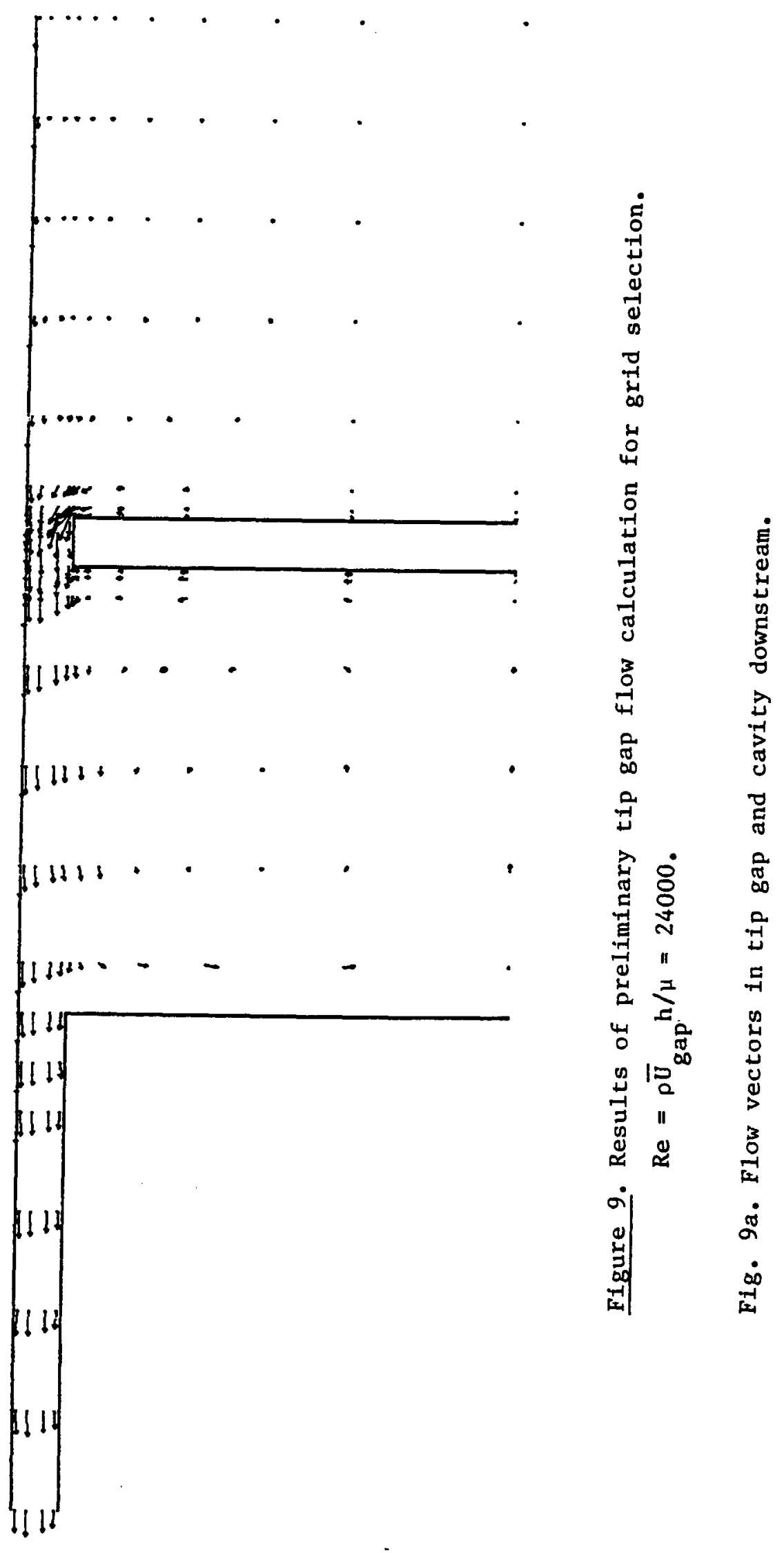




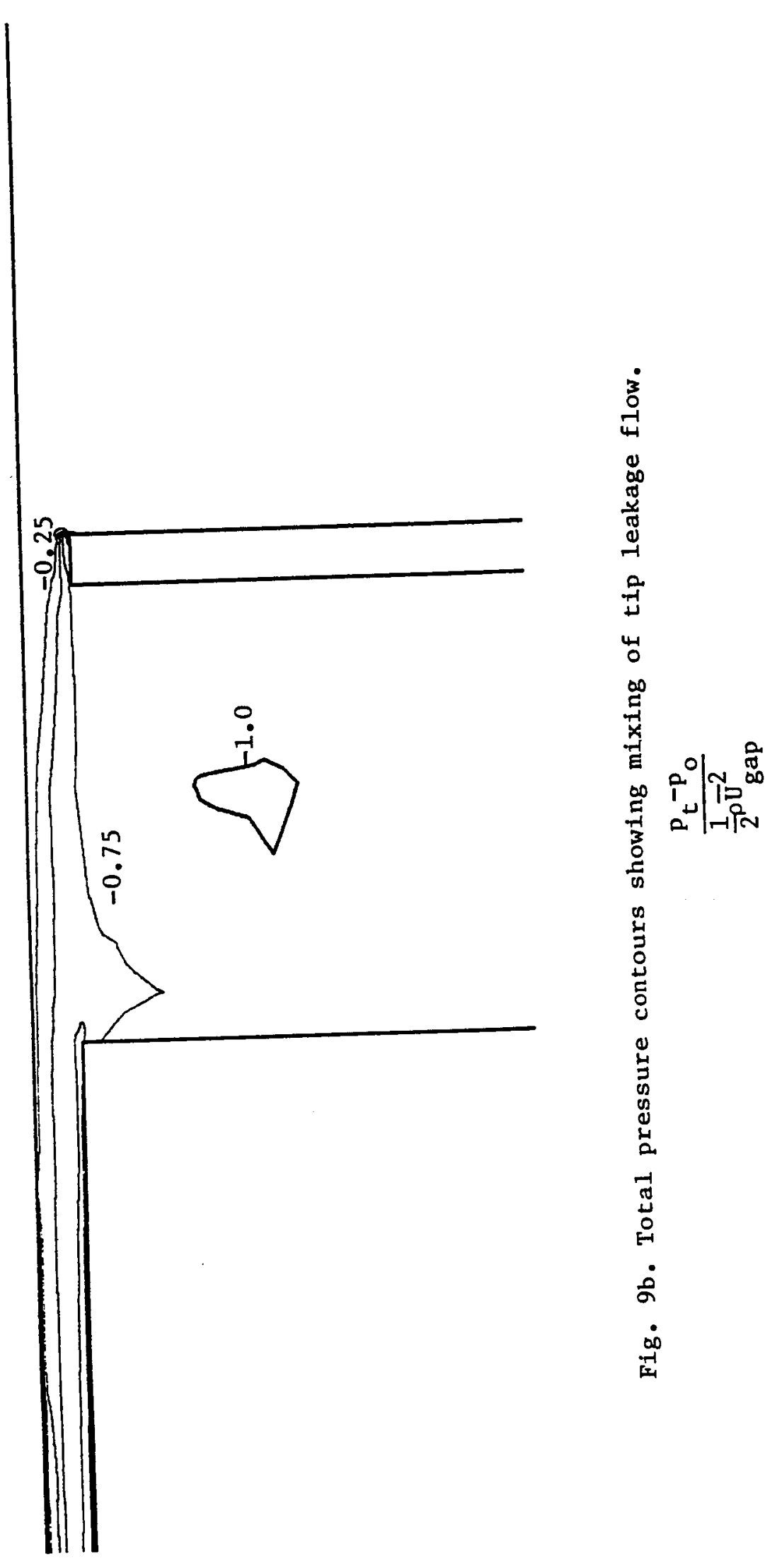


Fig. 9cl. Static pressure contours.

$\frac{p-p_{o}}{\frac{1}{2} \bar{U}_{\text {gap }}^{2}}+1$

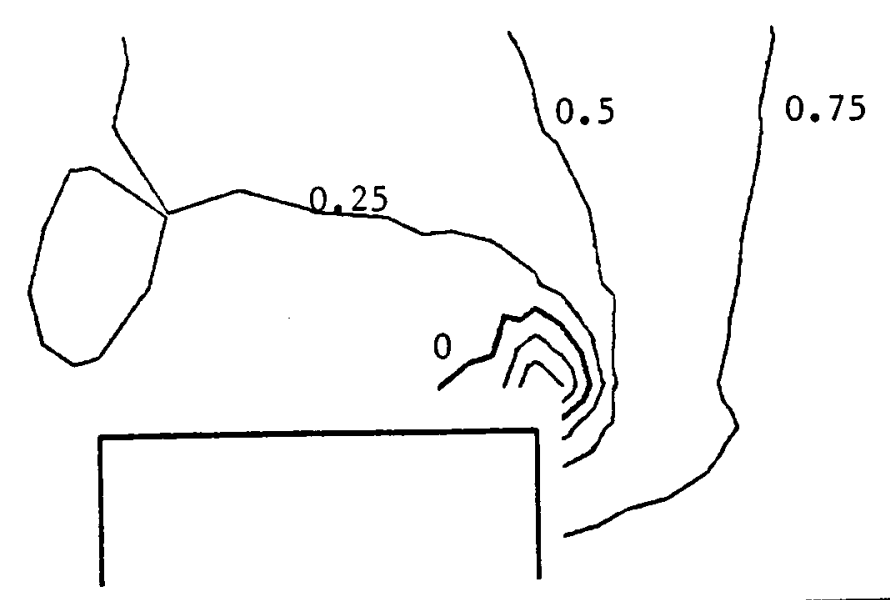

Fig. 9c2. Total

pressure contours.

$\frac{\mathrm{P}_{t}-\mathrm{P}_{\mathrm{o}}}{\frac{1}{2} \overline{\mathrm{U}}^{2}}$
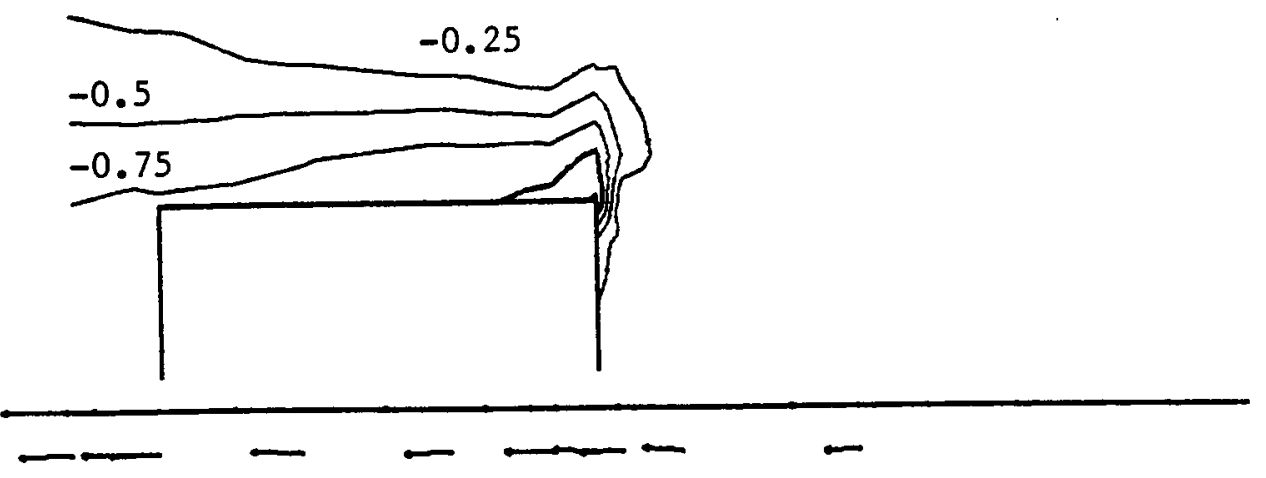

Fig. 9c3. Velocity vectors at the grid points.

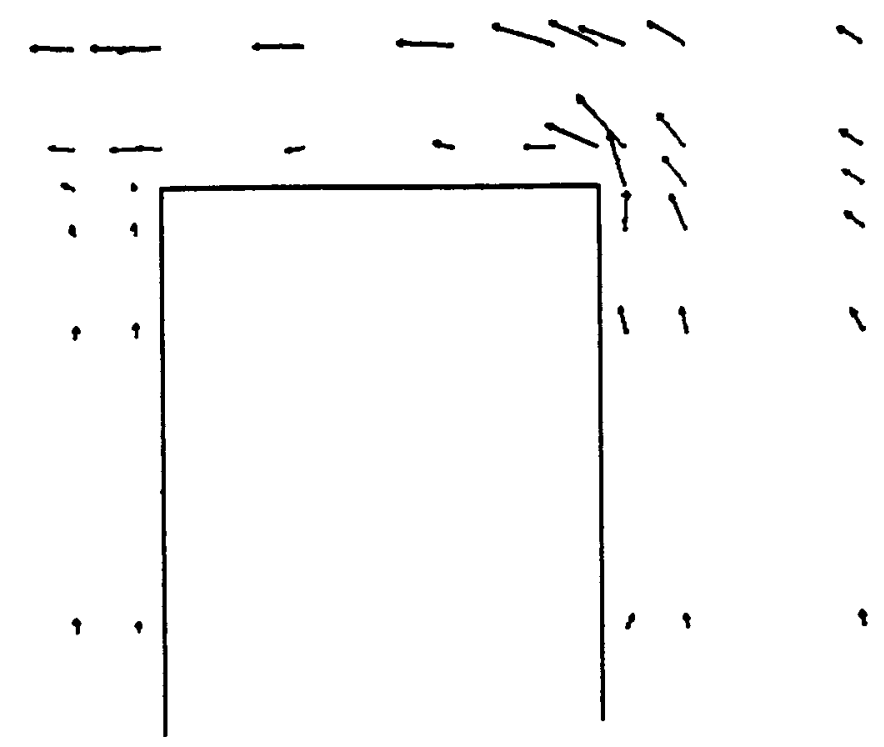

Fig. 9c. Flow detail over simulated blade tip. 


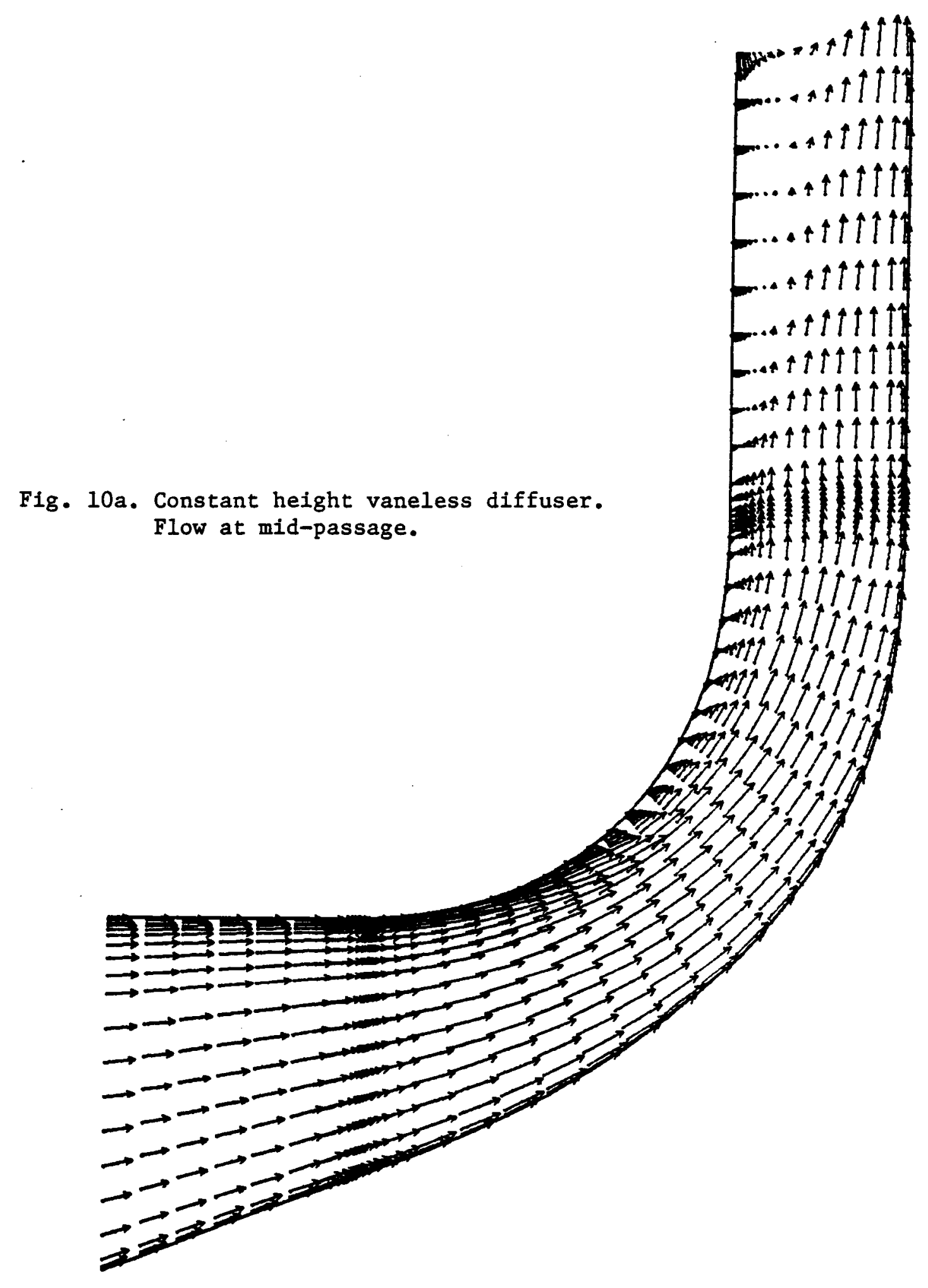

Figure 10. Meridional view of velocity vectors from the 3-D flow calculations. 


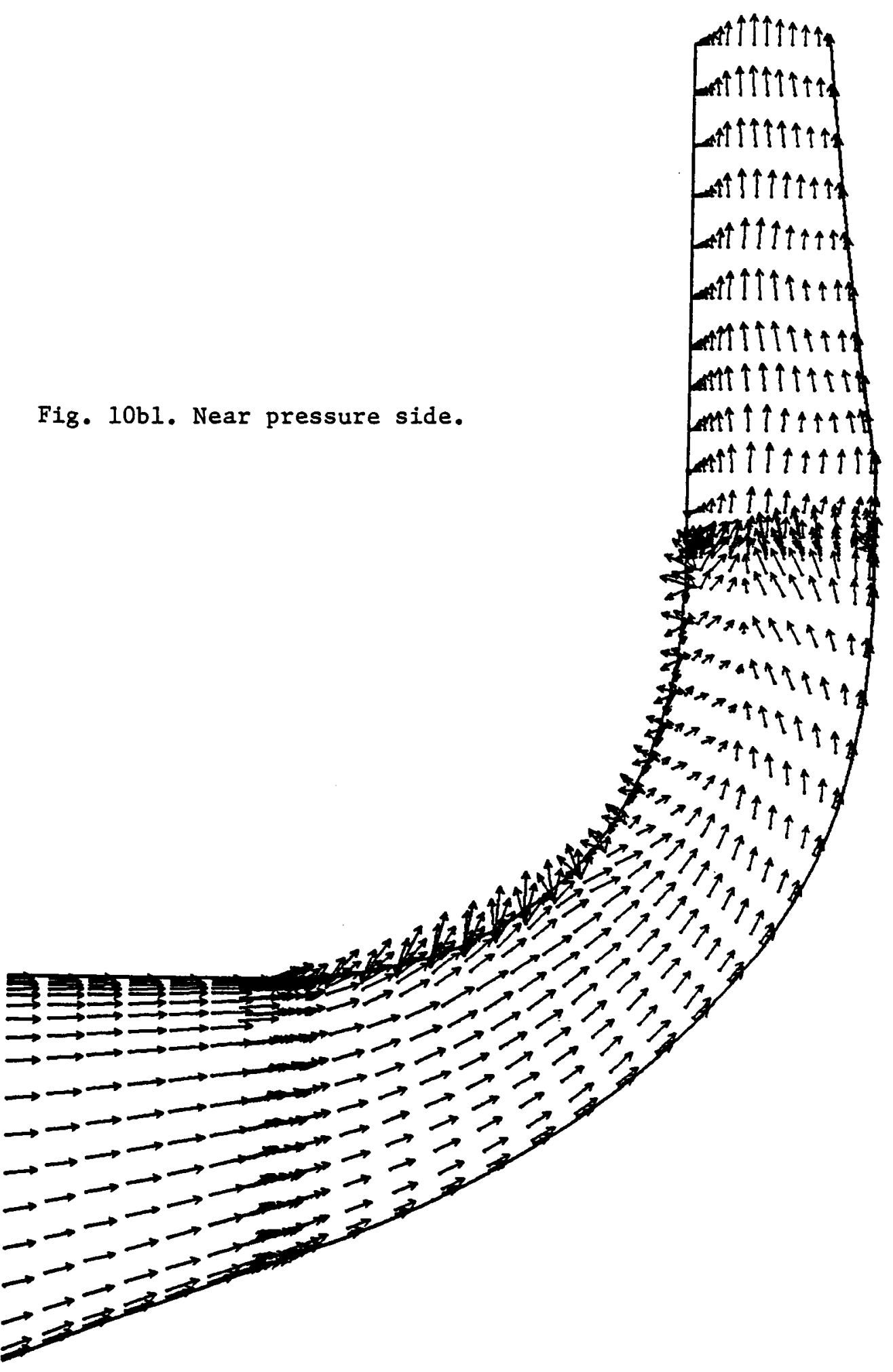

Fig. 10b. Constant area vaneless diffuser from $r / r_{2}=1.08$. 


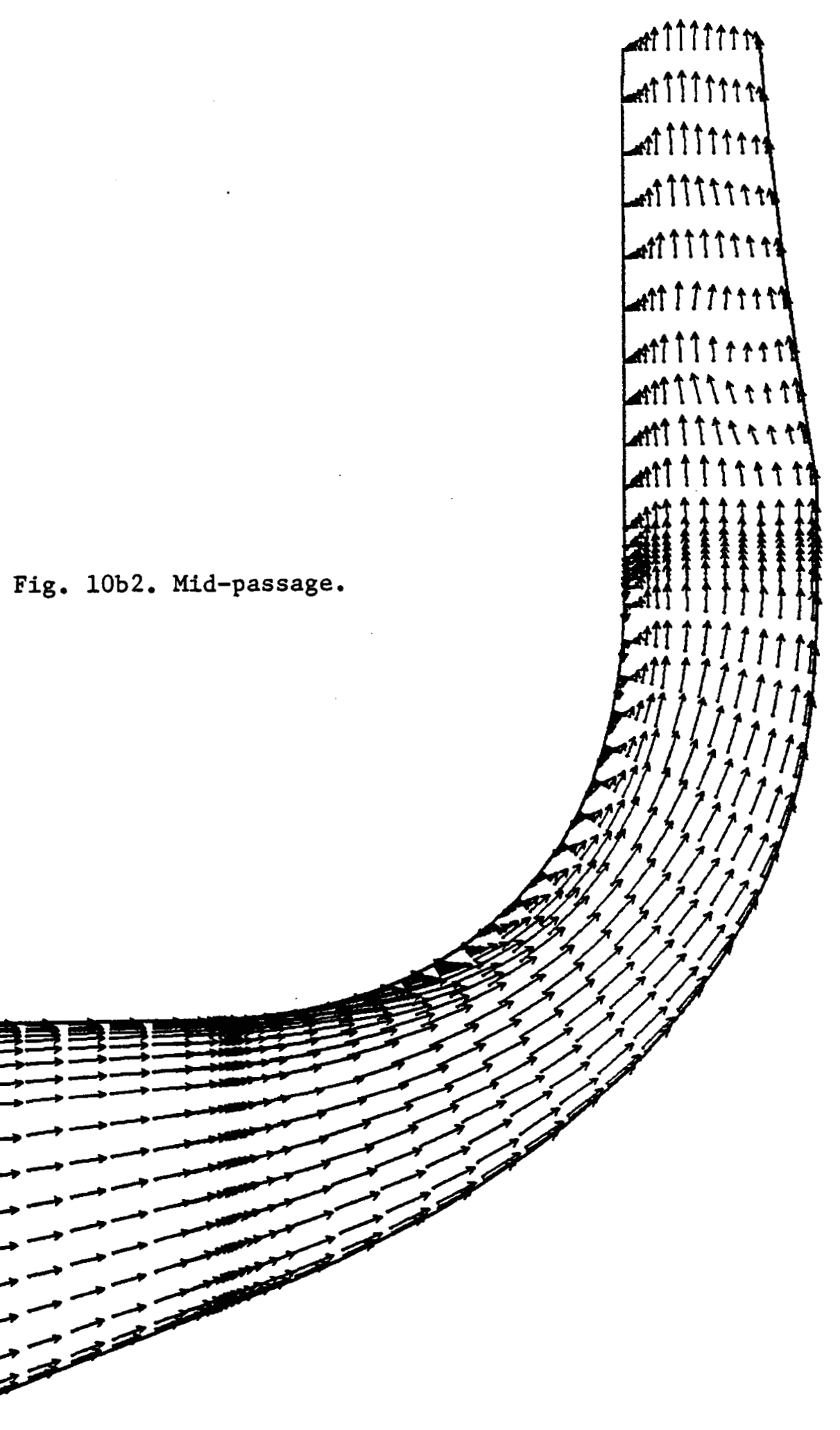




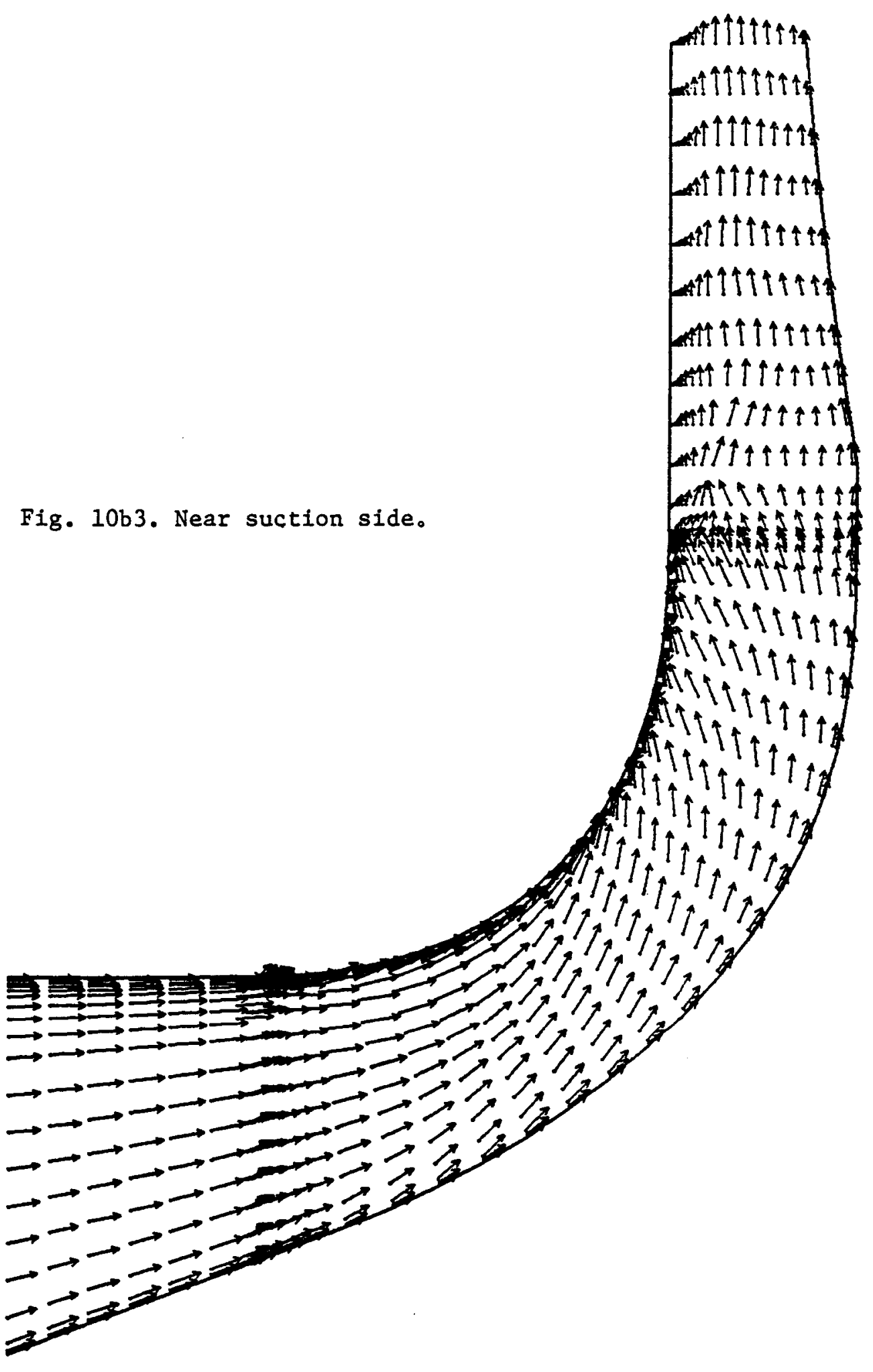




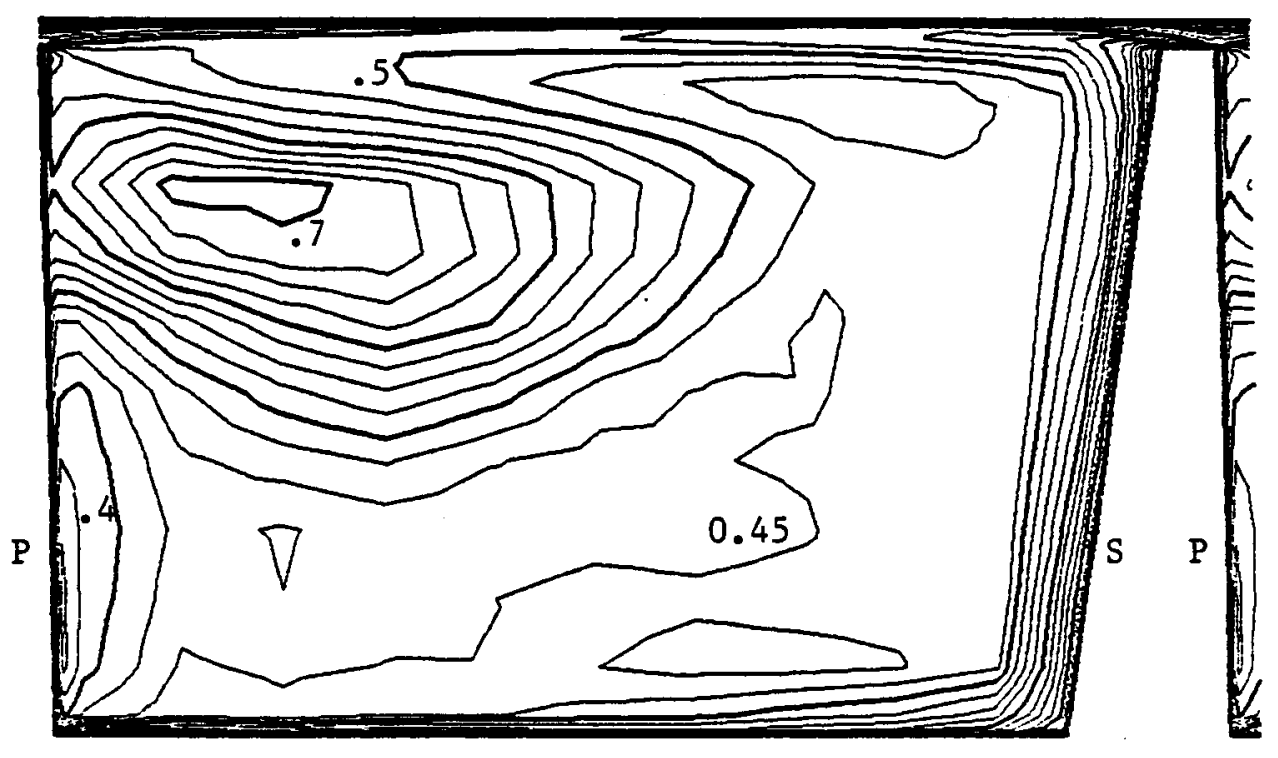

Figure 11. Contours of dimensionless absolute tangential velocity, $\mathrm{V}_{\theta 2} / \mathrm{U}_{2}$, in the exit plane of the impeller. Contour interval $=0.025$.

$P$, pressure side; $S$, suction side. 


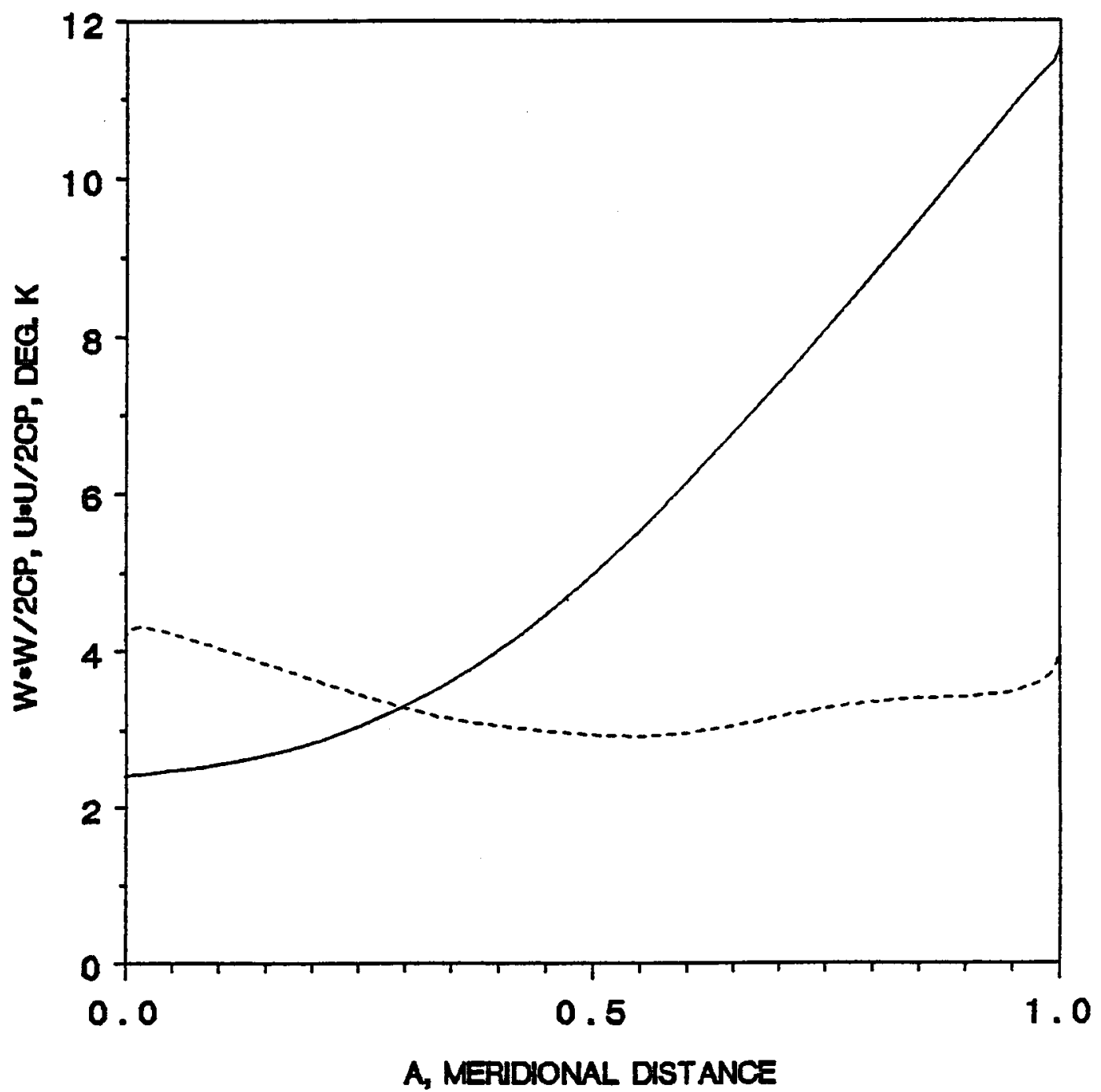

Figure 12. Contributions of centrifugal effects $(\longrightarrow$ and relative kinetic energy ( - - ) to temperature changes and work in the impeller. 


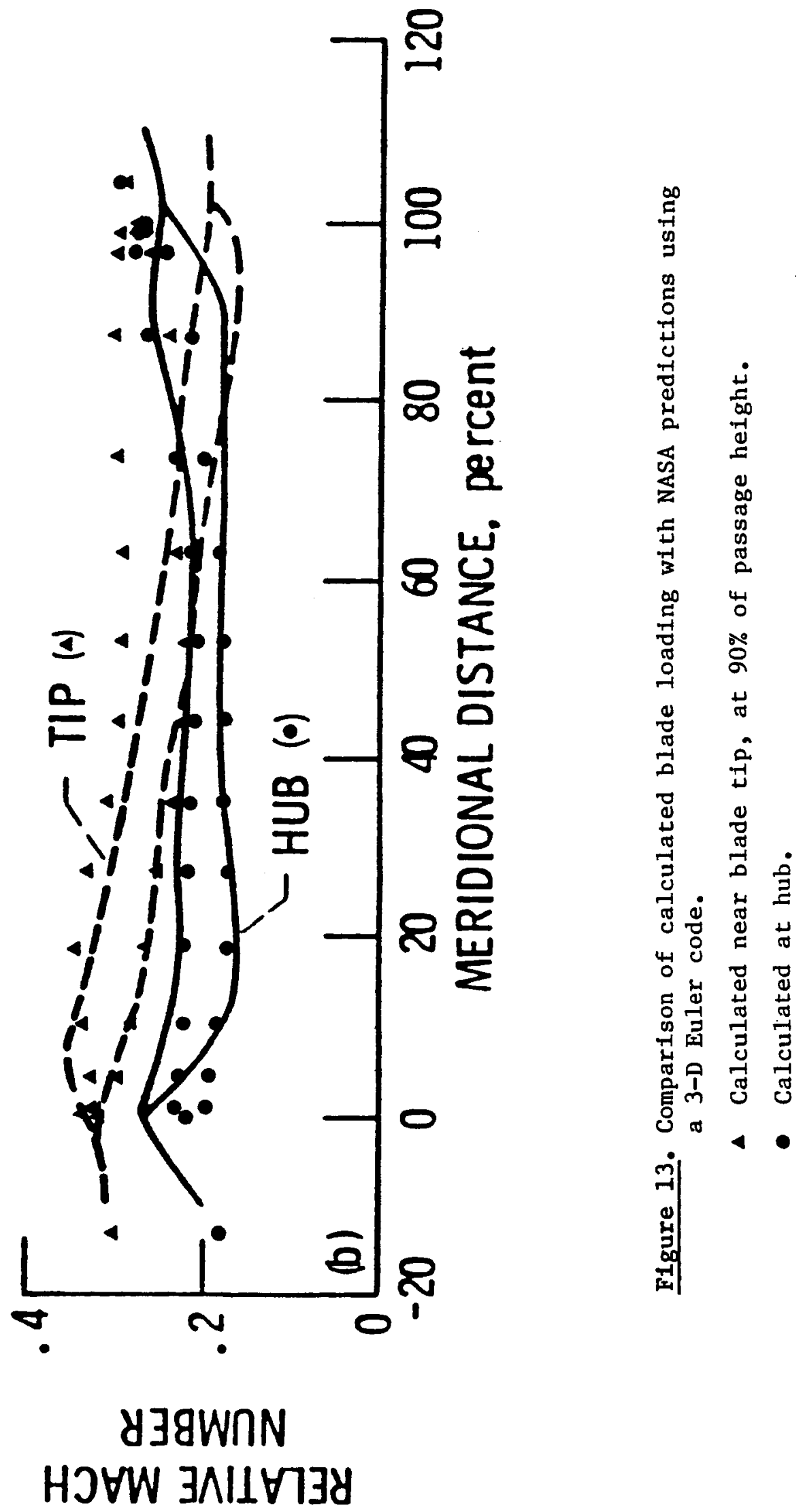




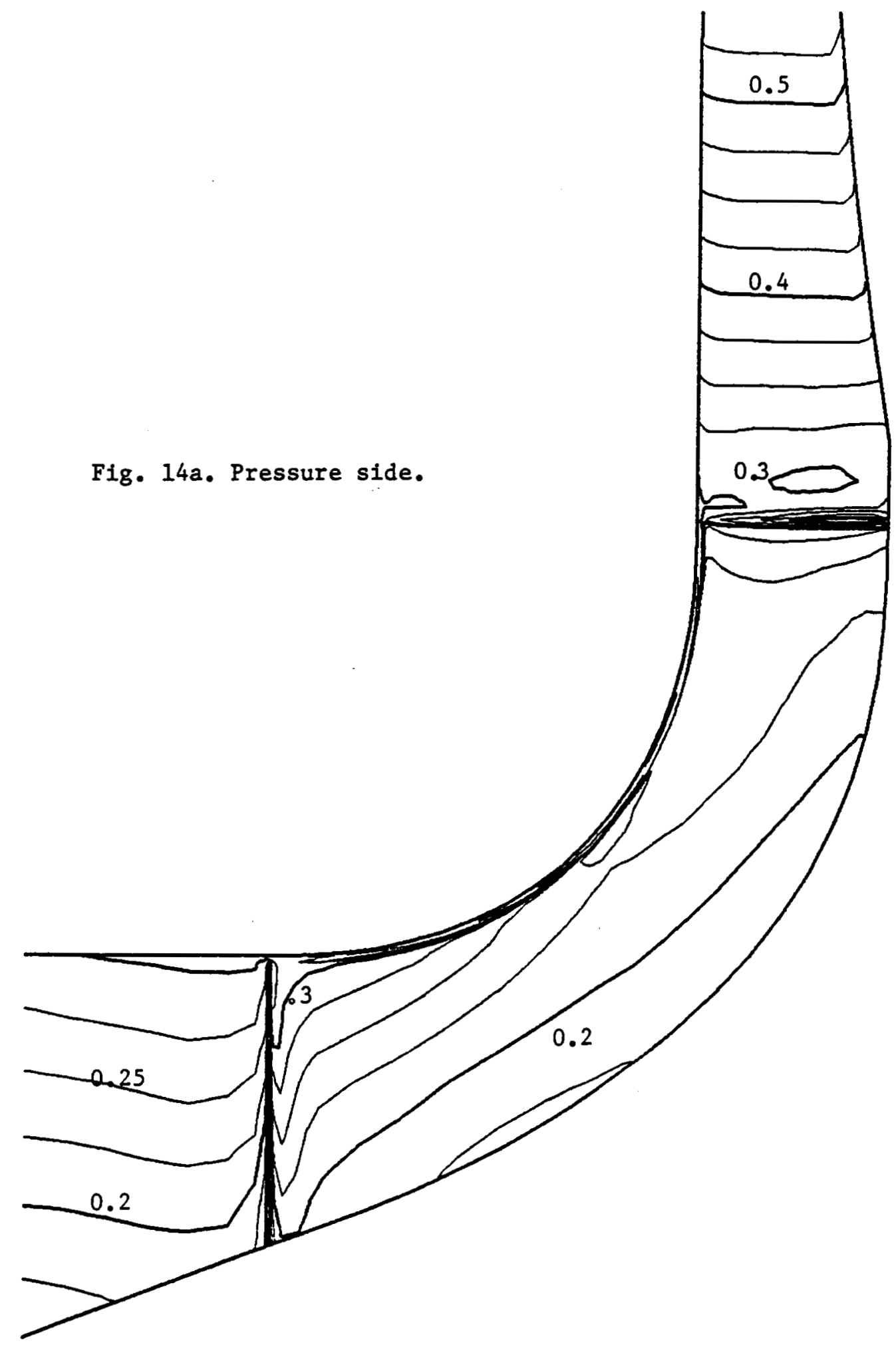

Figure 14. Blade surface reduced static pressures represented as isentropic relative Mach numbers. Contour interval $=0.025$ 


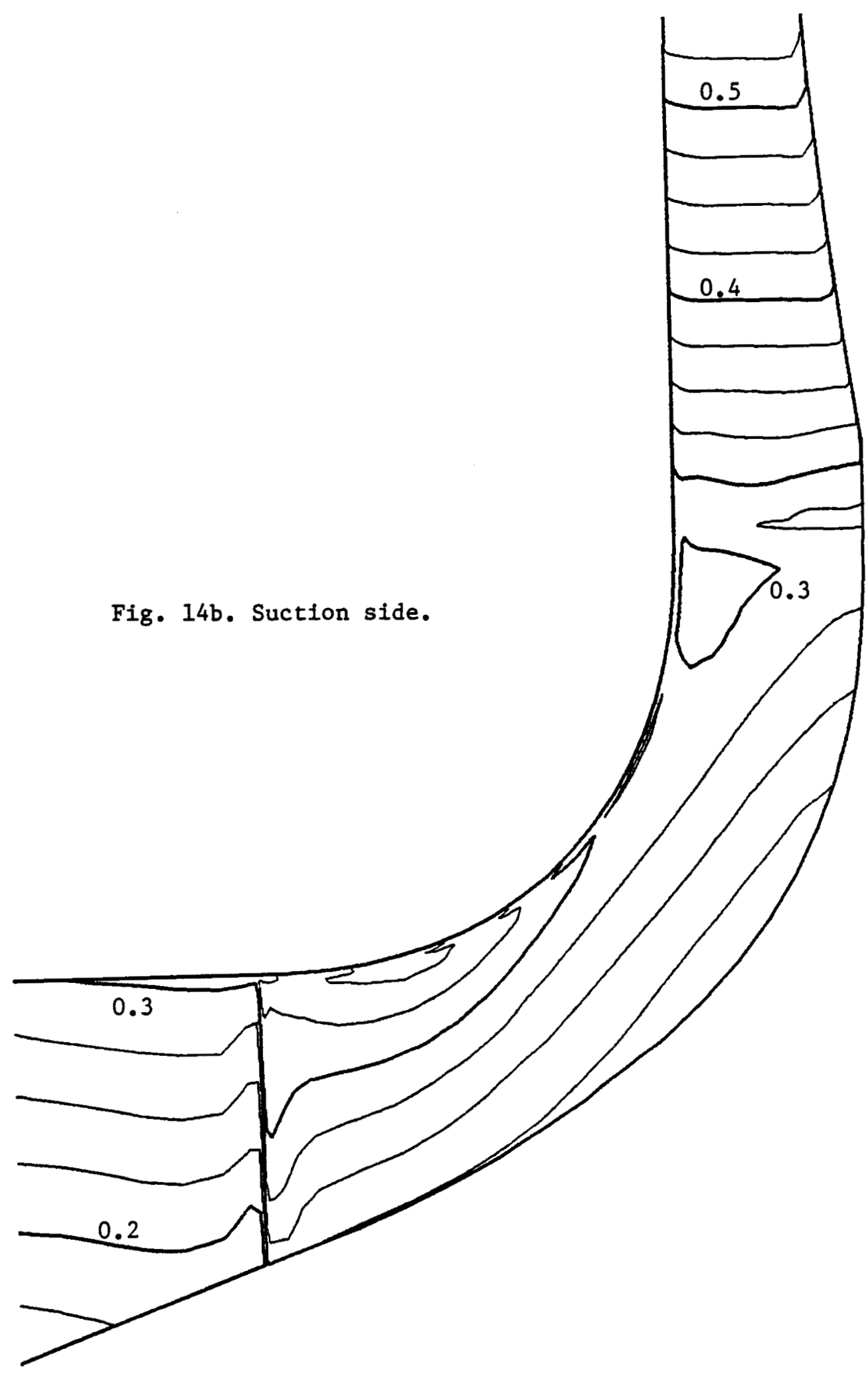




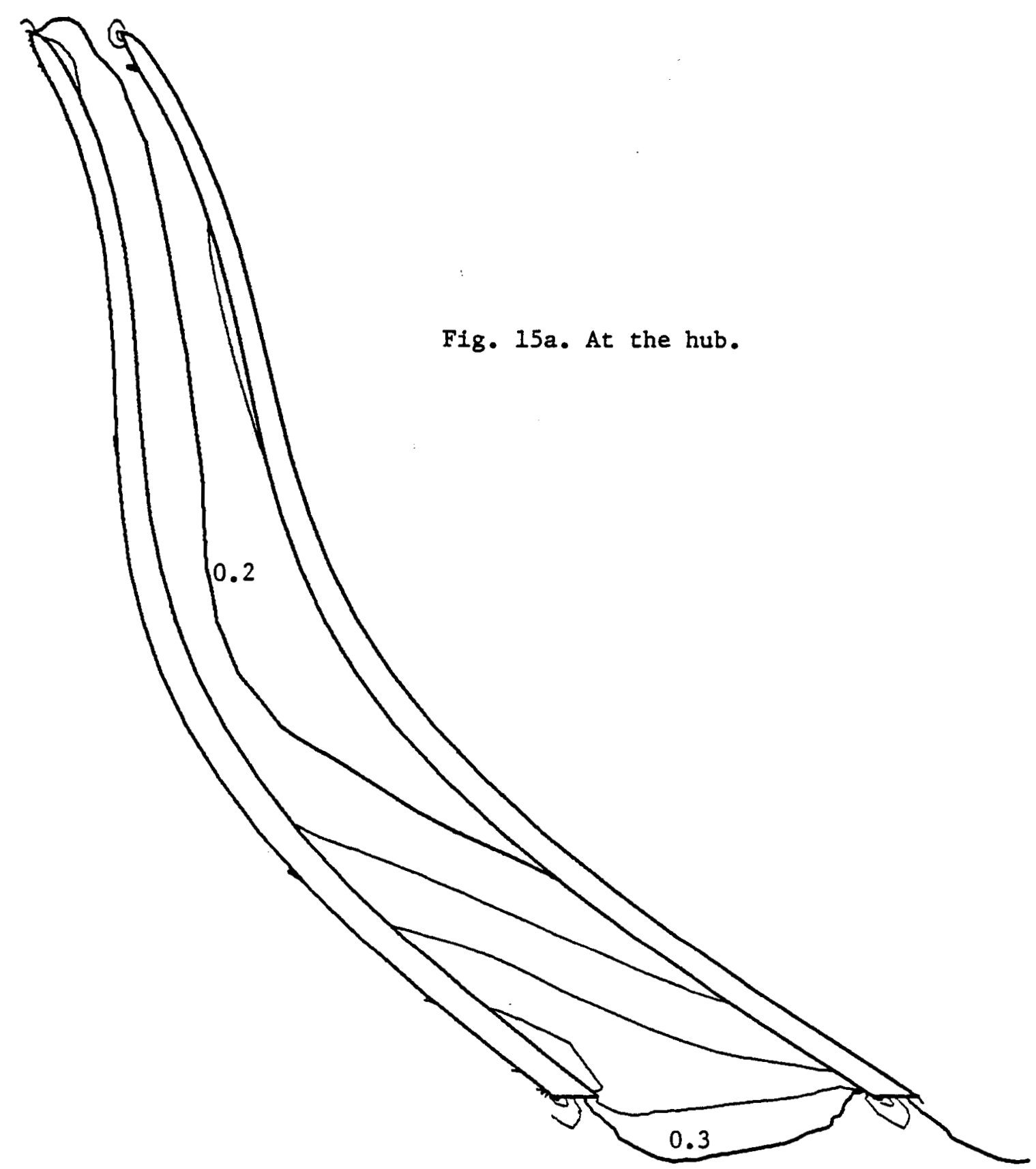

Figure 15. Distributions of reduced static pressure in blade-to-blade planes shown as contours of isentropic relative Mach number. 


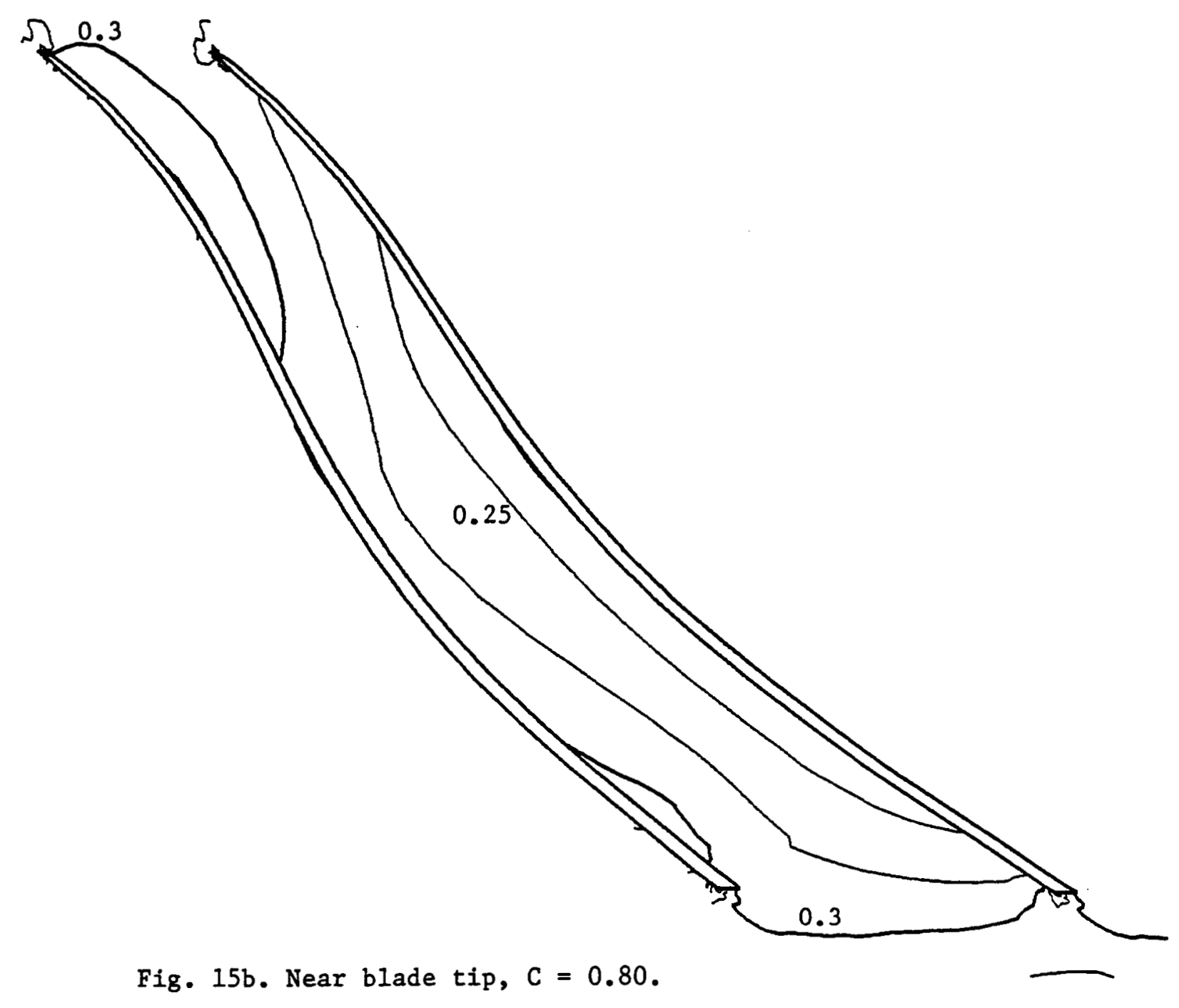




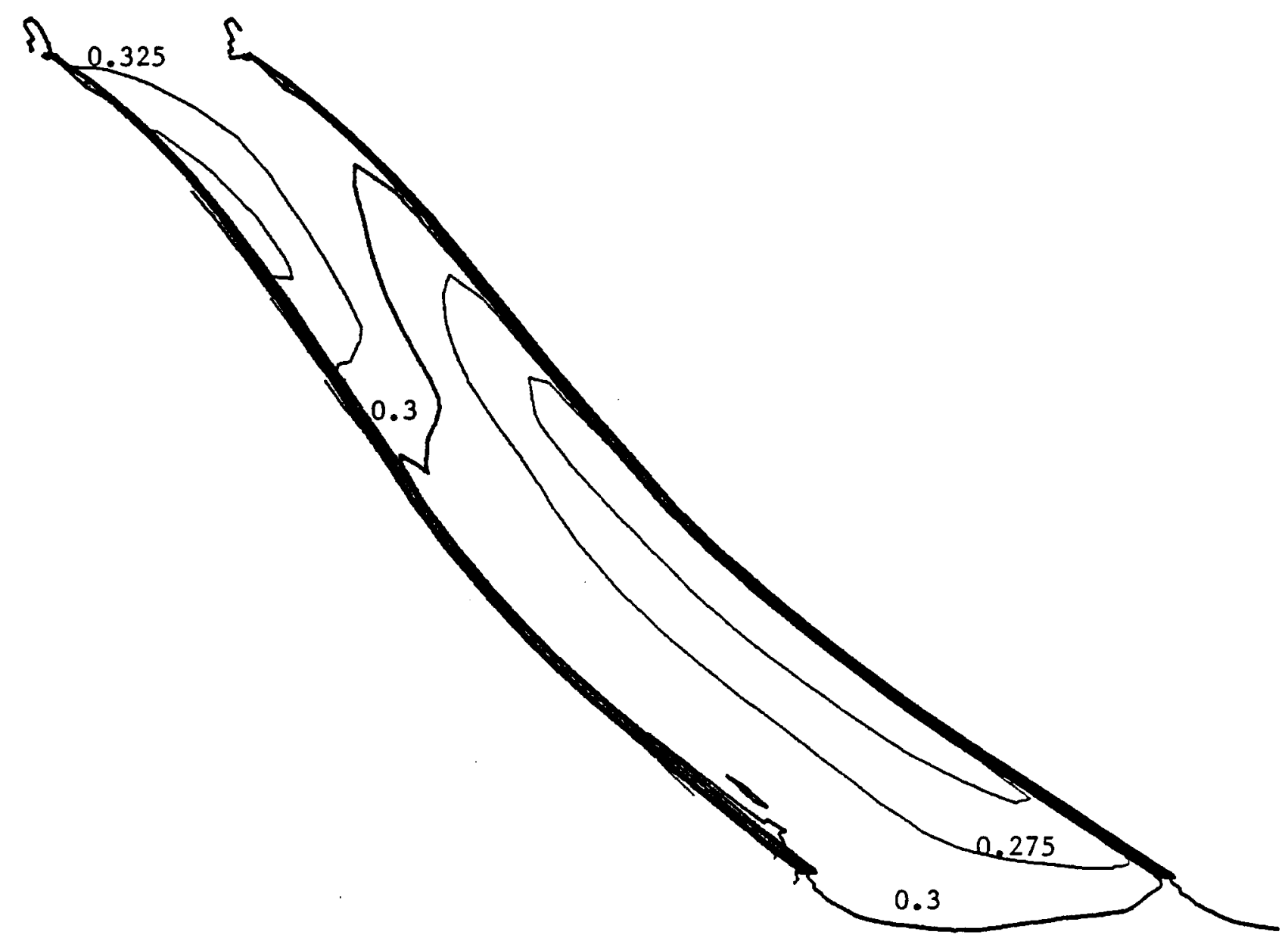

Fig. 15c. At height of blade tip, $C=0.97$. 


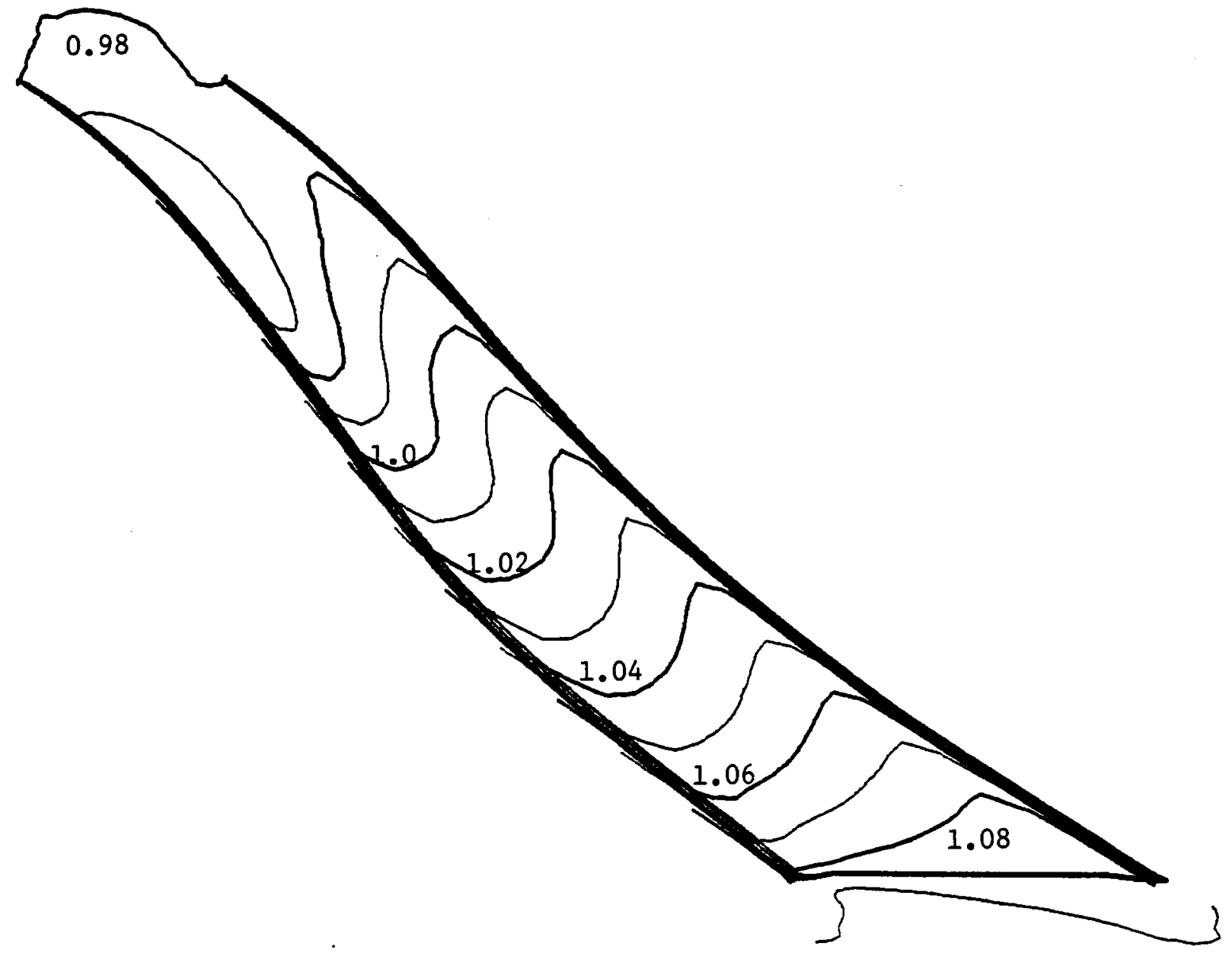

Figure 16. Shroud static pressure contours, $\mathrm{p} / \mathrm{p}_{\mathrm{o}}$, over the impeller passage. 


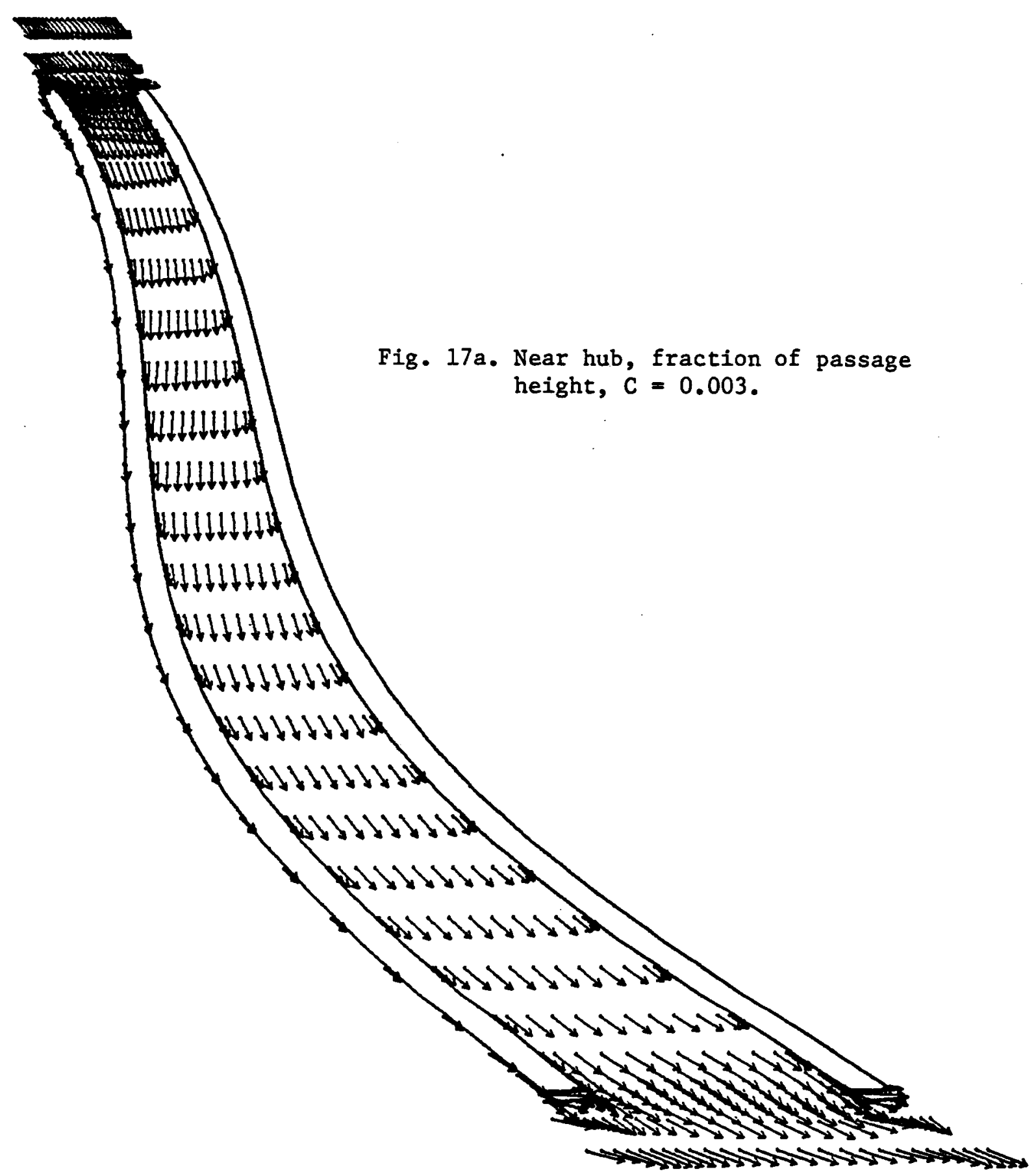

Figure 17. Throughflow velocity vectors projected onto blade-to-blade planes. 


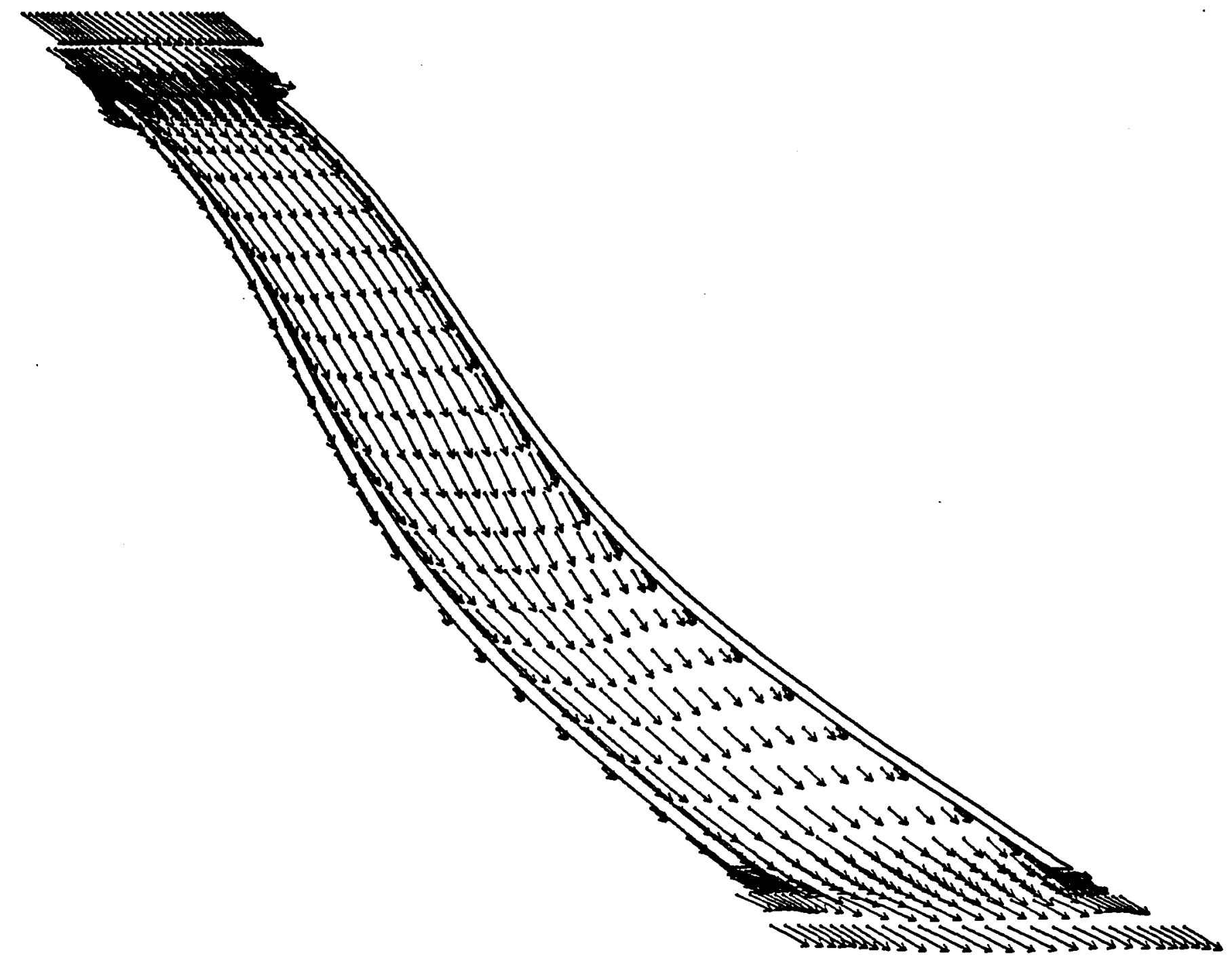

Fig. 17b. Near blade tip, $C=0.80$. 


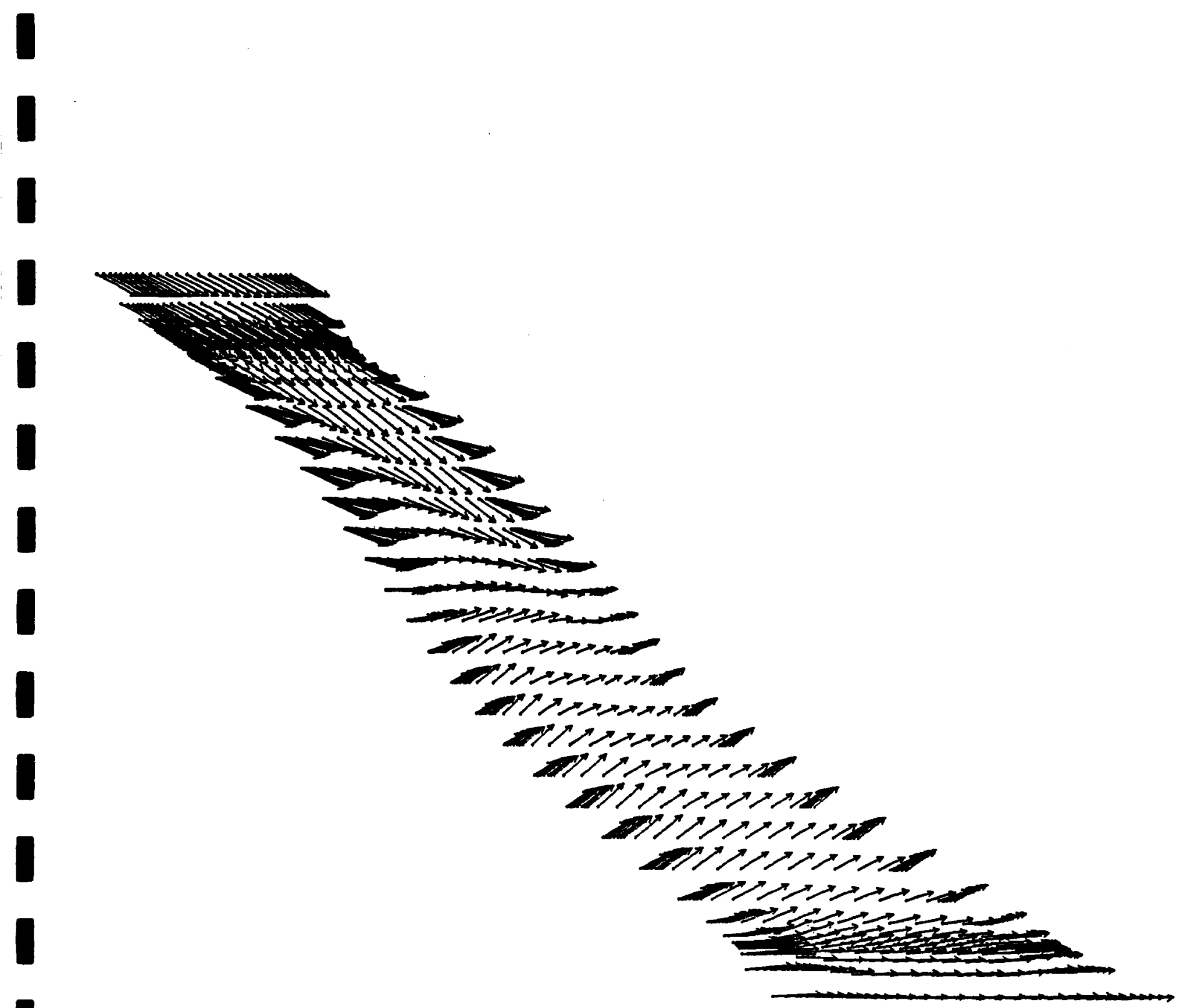

Fig. 17c. Near mid-height of blade tip gap, $C=0.99$. 

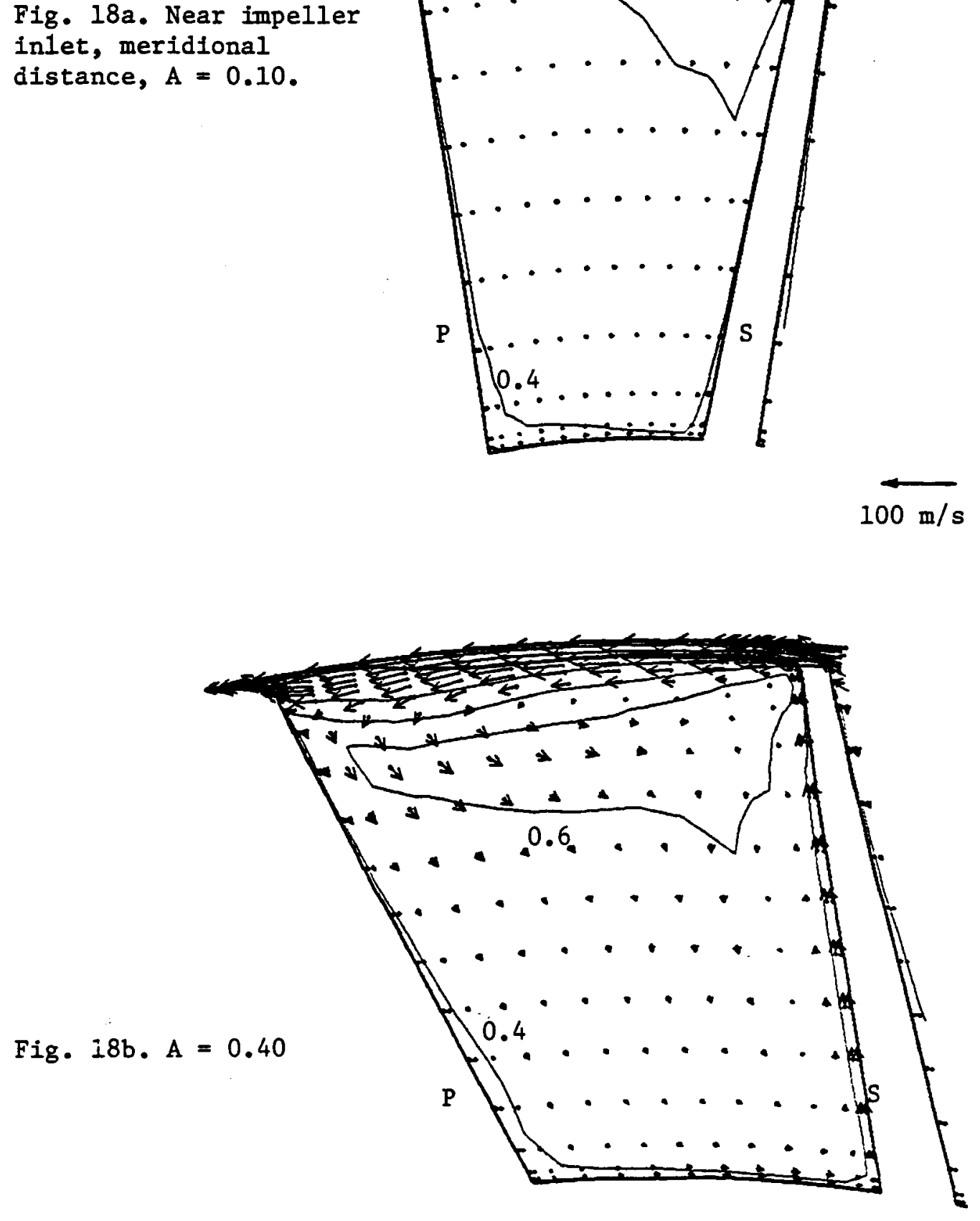

Figure 18. Calculated secondary velocity vectors on four crosssectional planes. Contours show distributions of primary velocity, $u^{p} / \mathrm{u}_{2}$ (contour interval $=0.2$ ). 


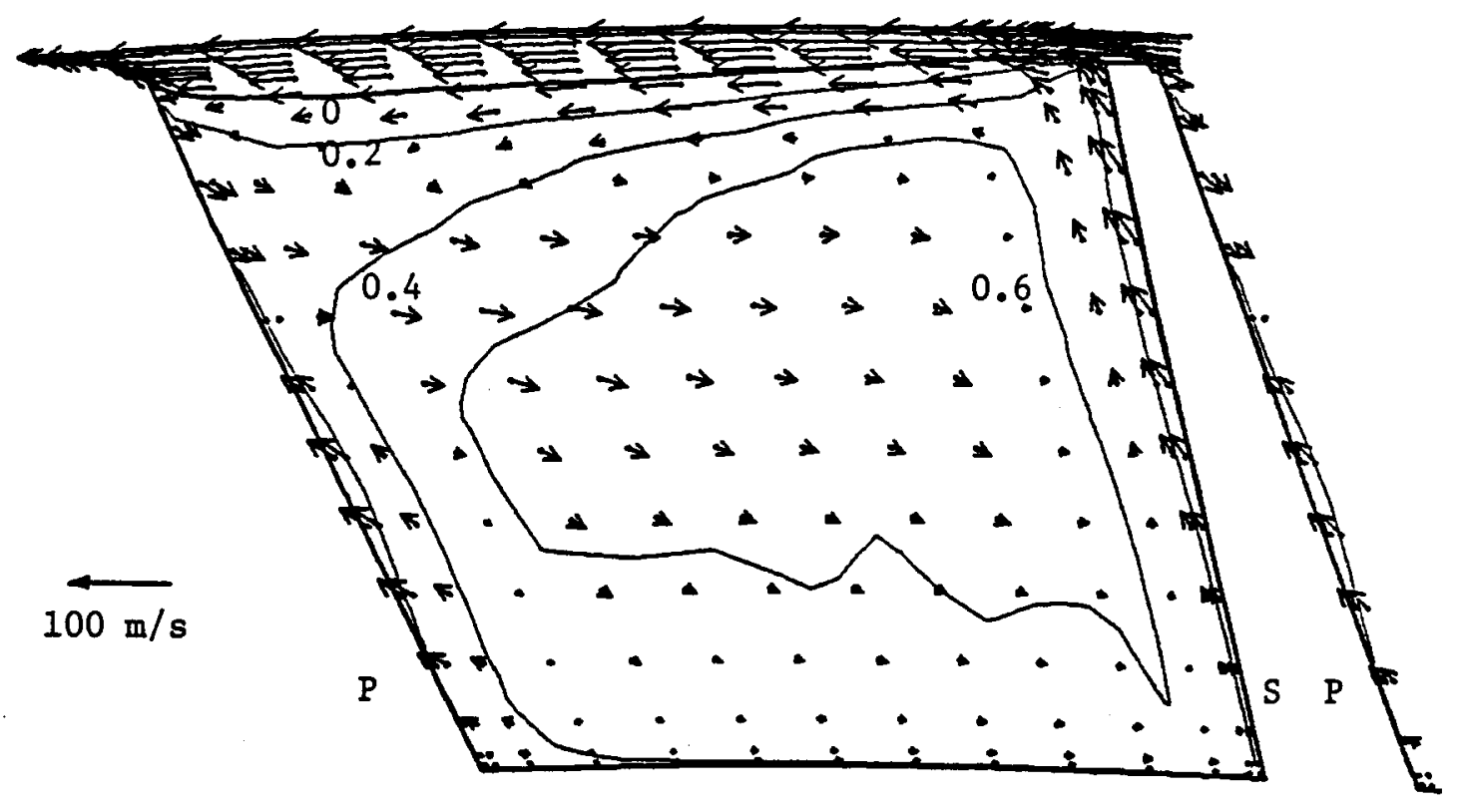

Fig. 18c. $A=0.74$

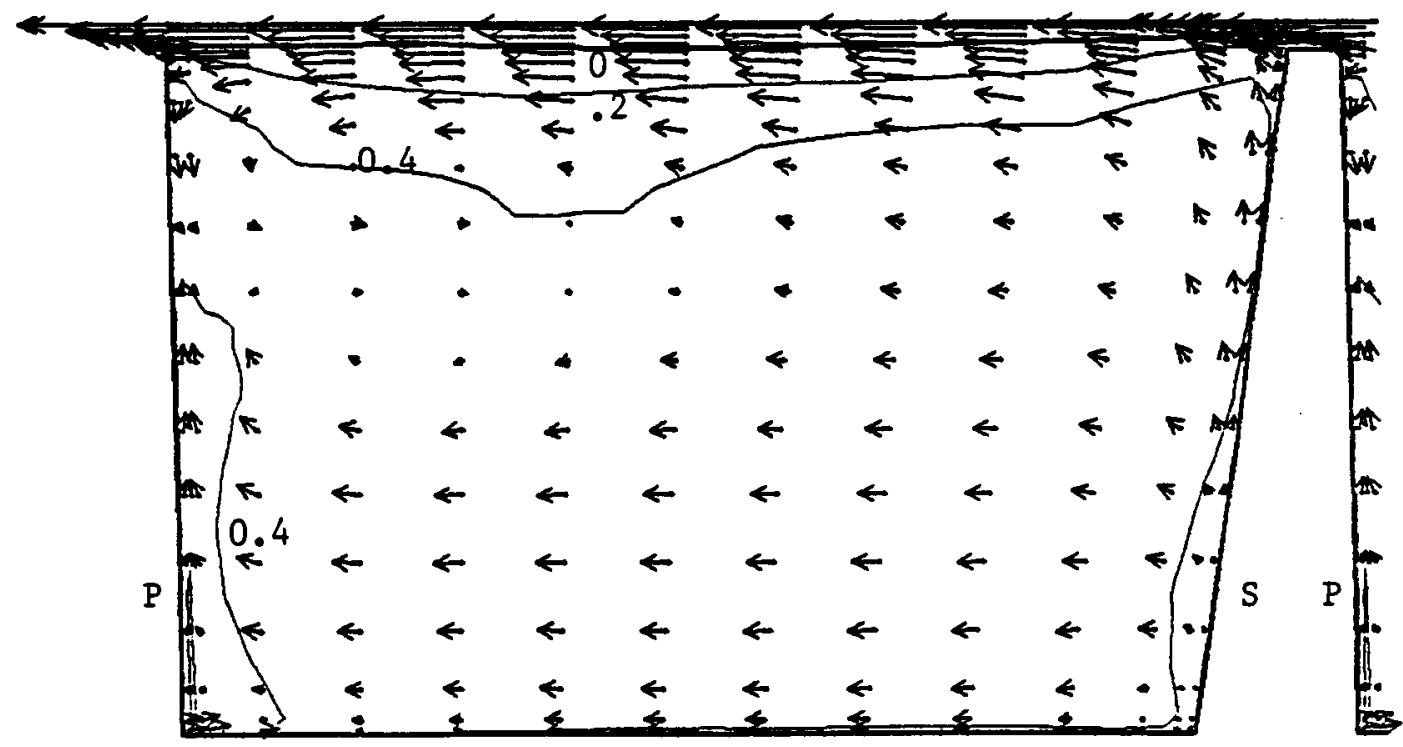

Fig. 18d. At impeller exit, $A=1.0$. 


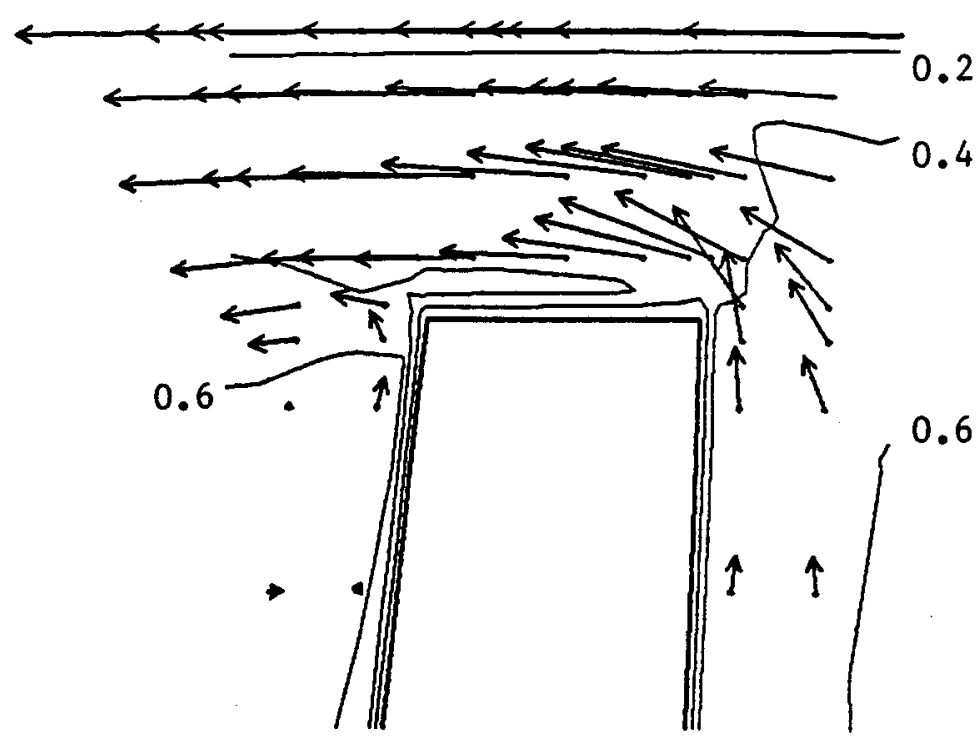

Fig. 19a. Near impeller inlet, meridional distance, $A=0.10$.

$$
100 \mathrm{~m} / \mathrm{s}
$$

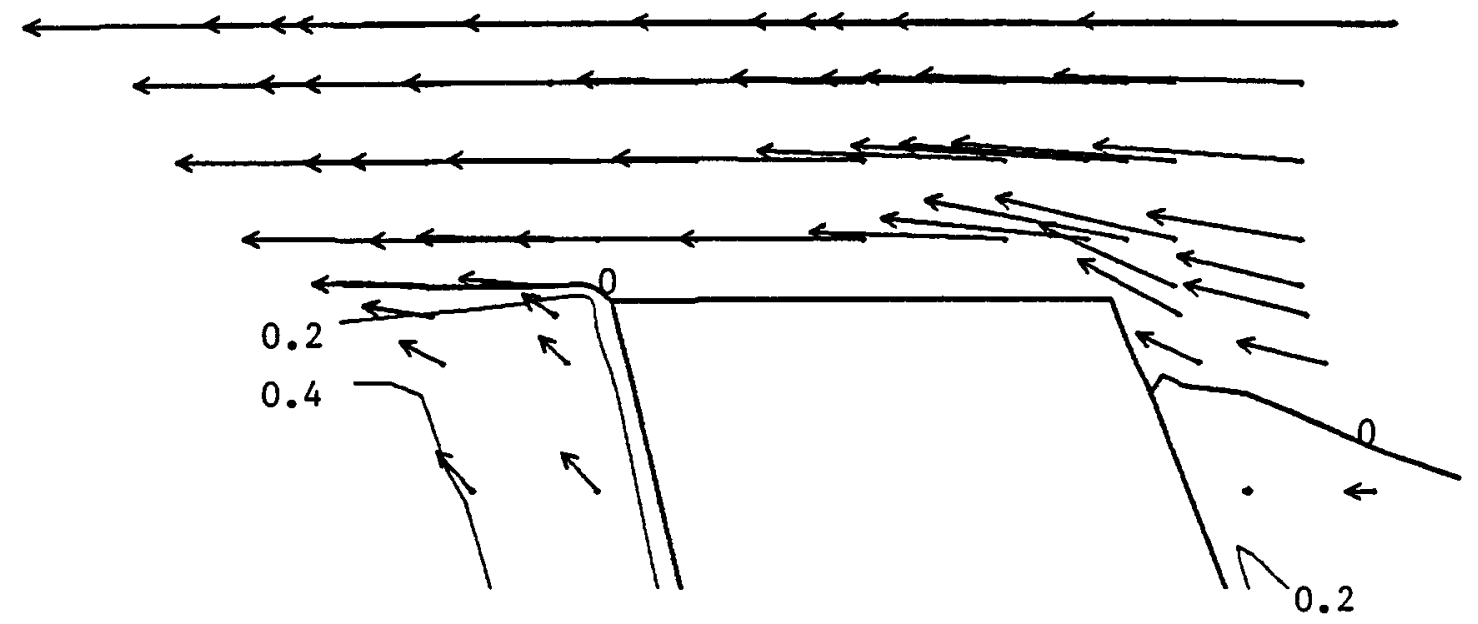

Fig. 19b. $A=0.74$

Figure 19. Calculated secondary velocity vectors and primary velocity contours for flow through the tip clearance gap. 


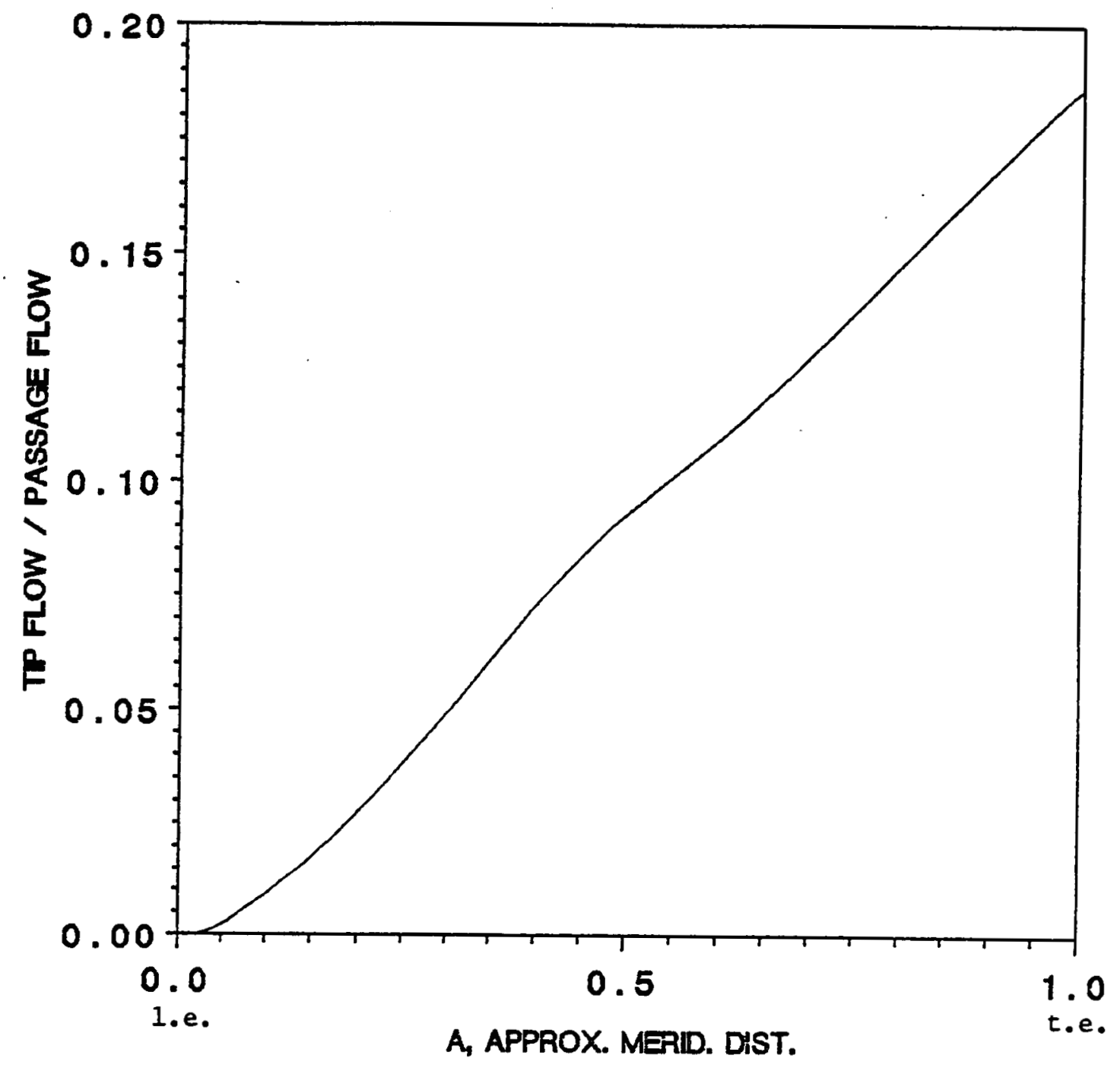

Figure 20. The cumulative fraction of the passage mass flow that passed through the tip gap. 


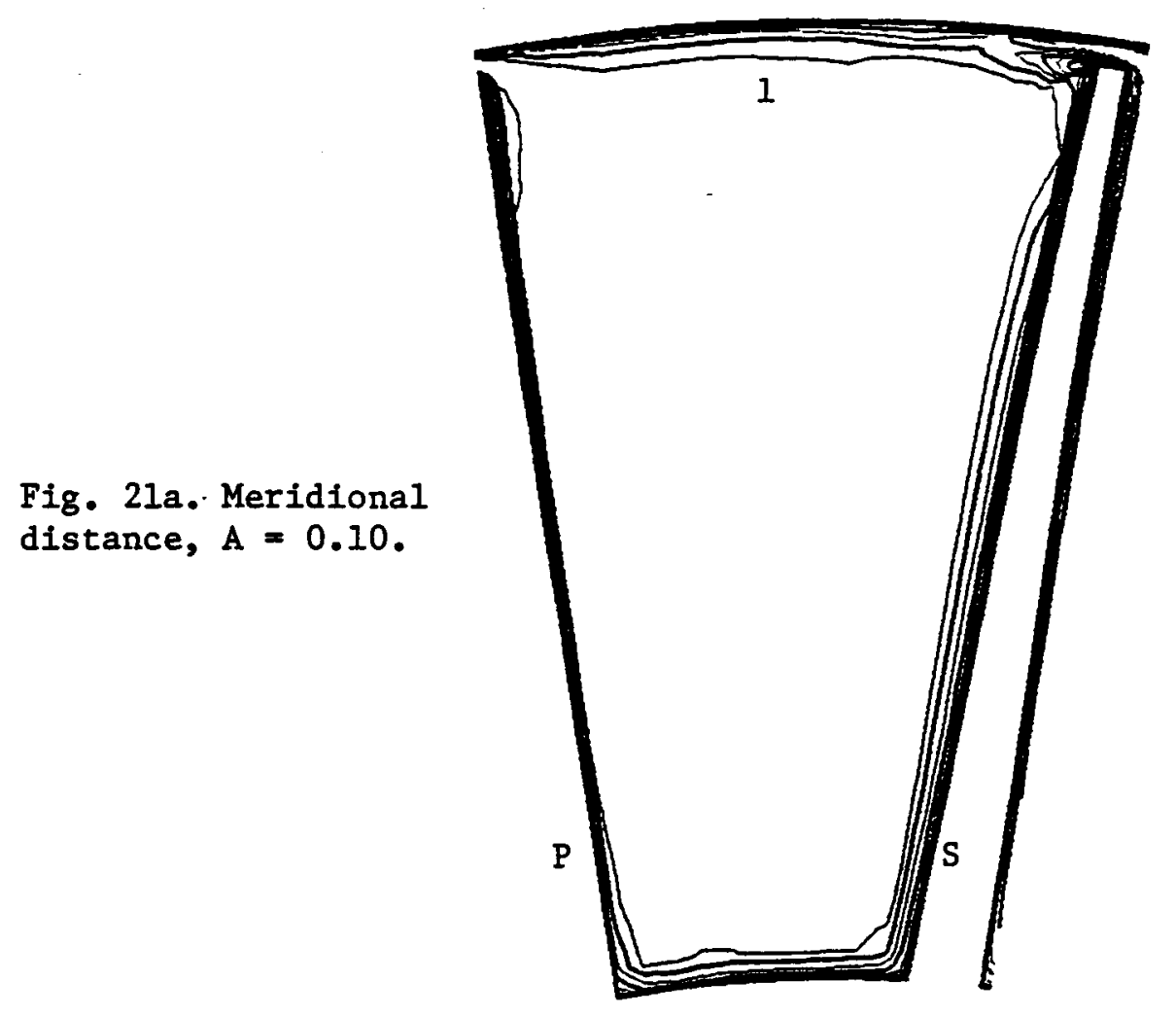

Fig. 21b. $A=0.40$.

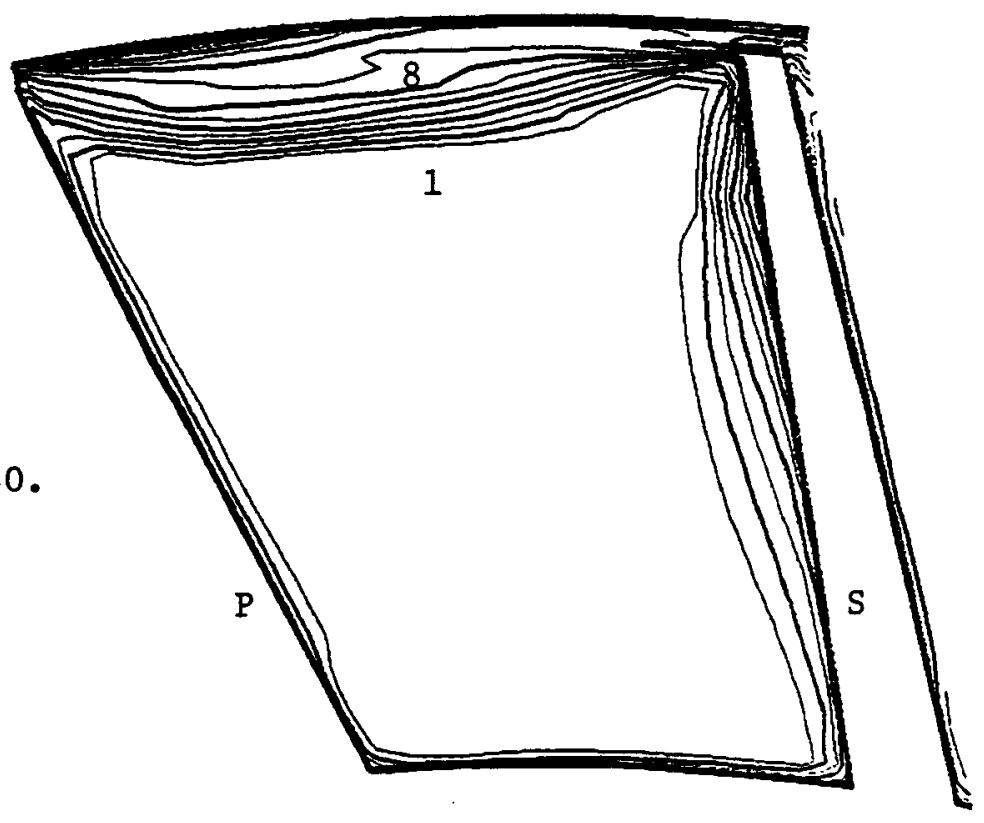

Figure 21. Distributions of entropy on cross-sectional planes through the impeller and the vaneless diffuser. Contour interval $=1.0 \mathrm{~J} / \mathrm{kgK}$. $\mathrm{P}$, pressure side; $\mathrm{S}$, suction side. 


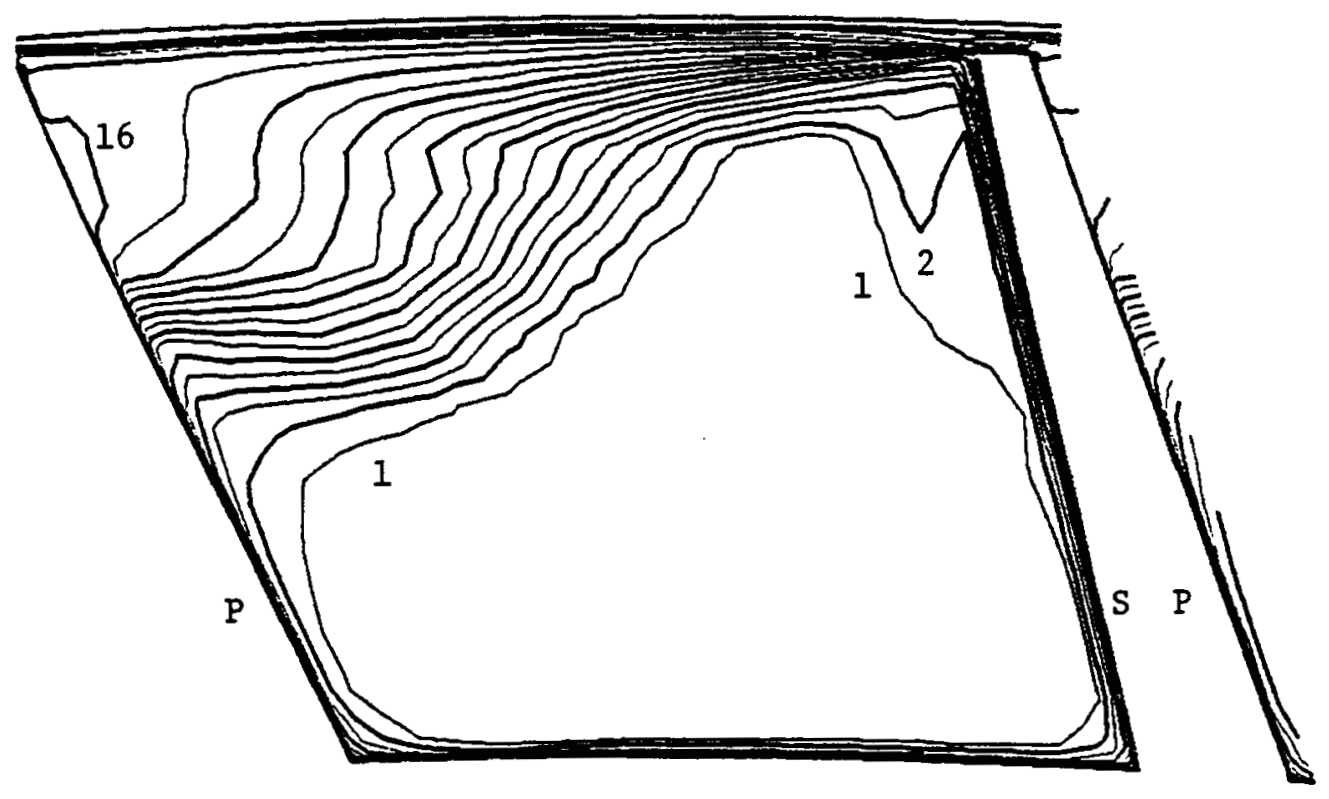

Fig. 21c. $A=0.74$.

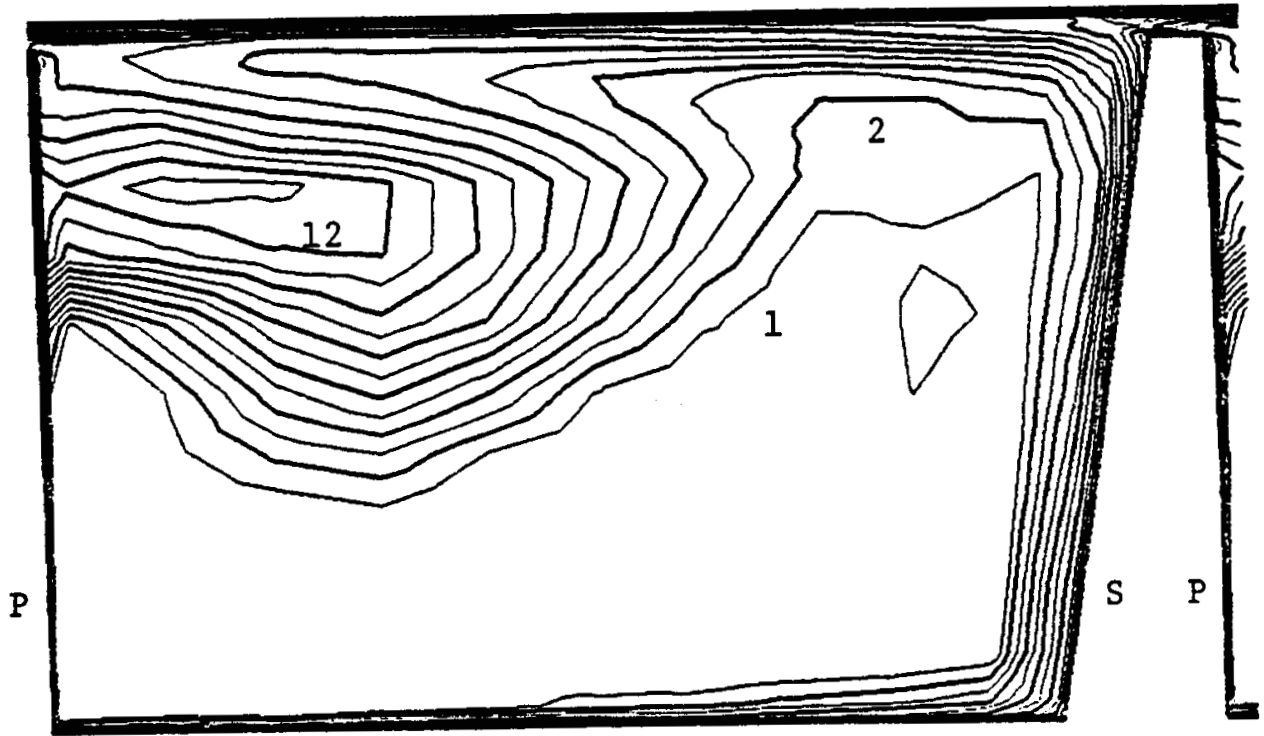

Fig. 2ld. $A=1.0$, impeller exit. 


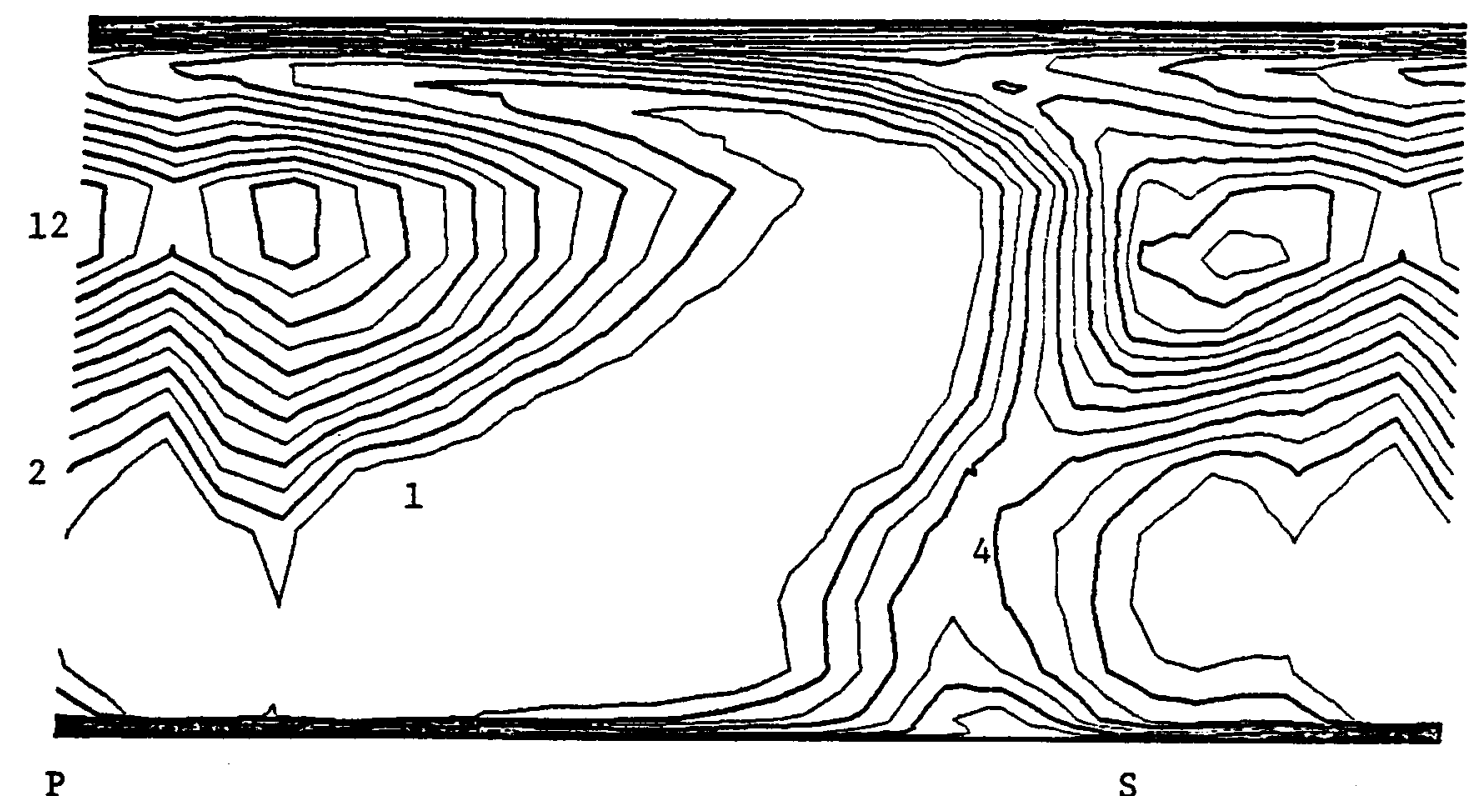

Fig. 21e. $r / r_{2}=1.04$, in vaneless diffuser.

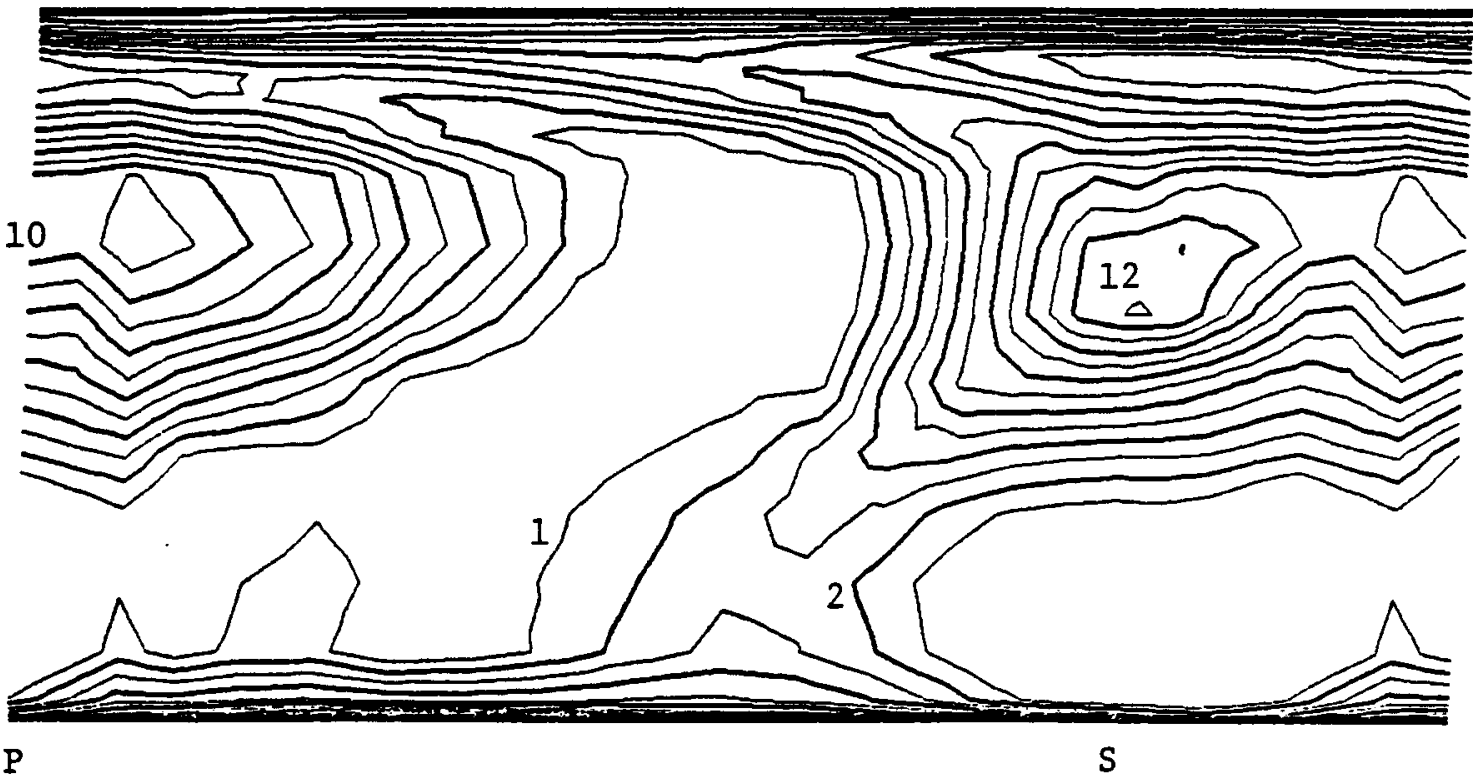

Fig. 2lf. $r / r_{2}=1.08$, in vaneless diffuser. 